\title{
LA DESTRUCCIÓN DEL MONUMENTAL CINEMA Y LA DEFENSA DEL PATRIMONIO ART DÉCO
}

Antonio Bravo Nieto

UNED-U niversidad de Málaga, España

El cine Monumental, construido con proyecto del arquitecto de Cartagena Lorenzo Ros, fue inaugurado en marzo de 1932 (Figs. 1, 2 y 3). La prensalocal de M elilla se hizo eco por entonces de la noticia en varias de sus ediciones. El Telegrama del Rif publicaba el 13 de marzo de 1932 un artículo titulado «En la presente semana se inaugura el monumental» y el 20 de marzo otro trabajo anunciaba «Se ha inaugurado el M onumental Cinema» (Fig. 4).

Pocos imaginaban que el fastuoso edificio que por entonces deslumbraba y dejaba perplejos a los melillenses por su monumentalidad y novedad, no llegaría a cumplir el medio siglo (Figs. 5 a 20). U na actuación inmobiliaria llevada a cabo a principios de los años ochenta acabaría con la principal obra del Art Déco melillense y una de las más destacadas construidas en este estilo en el ámbito español. En este pequeño trabajo, vamos a centrarnos en describir un aspecto muy poco conocido de este edificio, relatar el proceso que determinó su destrucción, y que se desarrolla fundamentalmente entre los años 1980 y 1981 (Fig. 21).

La característica crisis del cine sufrida desde los años setenta del siglo XX, hizo mella en las posibilidades empresariales del Cine M onumental y la familia Liarte, propietaria del inmueble, procedió a su venta después de un largo periodo de decadencia. La protección del patrimonio arquitectónico en M elilla a finales de los años setenta estaba todavía en una fase muy embrionaria y por entonces se estaban sentando las bases de lo que sería la catalogación definitiva del ensanche modernista de la ciudad. En esta línea el 9 de julio de 1979 se constituía la Comisión de Patrimonio de M elilla y la Dirección Provincial del Ministerio de Cultura intentaba realizar un primer inventario de edificios a proteger. ${ }^{1}$

1. En diciembre de 1978 se adjudicó la real ización de un inventario arquitectónico de Melilla. Los trabajos de catal ogación de edificios se realizaron en mayo de 1979 y el catálogo fue entregado en enero de 1980. El catálogo constaba realmente de 152 fichas, 68 fotografías y una memoria de 7 flios. 
Políticamente la ciudad estaba regida por el partido U nión del Centro Democrático, al ser elegido el 19 de abril de 1979 como alcalde de M elilla Rafael Ginel Cañamaque. El 22 de junio del mismo año había sido nombrado Comandante General de M elilla y Delegado del Gobierno J osé M aría Bourgón López Dóriga. ${ }^{2}$

Los trabajos de la recién creada Comisión de Patrimonio se encaminaron a conseguir que se incoaran expedientes para declarar el interés de los edificios más representativos de M elilla. El 11 de febrero se iniciaron las primeras gestiones y el 26 del mismo mes el M inisterio de Cultura solicitaba a la D elegación una relación de posibles edificios que pudieran ser declarados monumentos histórico-artísticos. En respuesta a esta iniciativa, la Comisión remitía el 11 de marzo al Director General una relación de 25 edificios de marcado interés para iniciar su protección, entre los que ya se encontraba el Cine M onumental.

Sin embargo, la venta del Cine M onumental era ya un hecho que se estaba negociando en esos momentos, y en julio de 1980 su nuevo propietario, Ashok J hamandas Lalchandani requería los ser vicios del arquitecto J osé María Bourgón de Izarra para convertir el antiguo cine en un edificio de locales comerciales. El día 4 de agosto de 1980, el propietario remitía un escrito al Presidente de la Comisión del Patrimonio Histórico Artístico de M elilla junto al estudio previo de la reforma del cine M onumental. La idea inicial era que se aprobara este estudio, para desarrollar el posterior proyecto y conseguir la preceptiva licencia de obras. Por su parte, la propiedad también elevaba la misma documentación al Ayuntamiento de M elilla, con los planos de la reforma. El Ayuntamiento contestaba el 1 de octubre señalando que el Servicio de Arquitectura estimaba correcta la modificación y que cumplía la normativa del plan general.

Por su parte, la Comisión de Patrimonio se reunía el 6 de octubre del mismo año $1980^{3}$ en una reunión que final mente resultaría trascendental para la suerte del

Por el mismo tiempo, en diciembre de 1979, LucianoTejedor M ata realizaba al gunos de los primeros inventarios conocidos de los edificios más importantes de M elilla, que él catalogaba como modernistas, historicistas y racionalistas.

2. Durante este periodo, el Comandante General de la ciudad ejercía al mismo tiempo el cargo de Delegado del Gobierno, y como tal, jefe superior del Delegado del M inisterio de Cultura en M elilla. José M aría Bourgón y López Dóriga fue nombrado el 22 de junio de 1979 y cesó el 14 de agosto de 1981. Anteriormente había desempeñado el cargo de Director del CESID.

3. Acta $n .-5$ del día 6 de octubre de 1980. Asistieron a esta comisión el presidente y delegado de Cultura Enrique Ibáñez Narváez, Francisco M ir Berlanga como representante de las corporaciones locales, Joaquín Rodríguez Puget como representante de ServiciosTécnicos, Alfonso Álvarez 
M onumental. Por un lado se estudió el inicio de los trámites de incoación de 10 de los 25 edificios propuestos, pero el tema fundamental fue el estudio del anteproyecto de reforma para su aprobación o denegación. Después de un largo debate los reunidos aprobarían el anteproyecto presentado, siendo Luciano Tejedor M ata la única persona de la Comisión que votó en contra del anteproyecto, al considerar que no se respetaban ni los interiores ni la fachada lateral. La Comisión por entonces se preocupó más por mantener la imagen externa del edificio que conservar sus interiores, por lo que realmente el futuro del cine quedó sentenciado en esta fecha. ${ }^{4}$ C uriosamente, en la misma reunión se acordó iniciar el expediente de declaración de los diez edificios más significativos de la ciudad, siendo uno de ellos el referido M onumental.

En enero de 1981 el arquitecto J ose M aría Bourgon de Izarra daba forma definitivamente al «Proyecto de reforma del cine M onumental, para su conversión en edificio de oficinas y locales comerciales, emplazado en las calles A. de Mera y Ejercito Español, en M elilla, para D. Ashok Jhamandas Lalchandani». El proyecto estaba compuesto de memoria, presupuesto básico y planos, numerados del 1 al 7 que correspondían al estado actual y del 8 a 23 al proyecto básico. El arquitecto, que justificaba el denominado proyecto de reforma, señalaba al hablar de la obra de 1932 que la fachada lateral había sido «tratada de forma secundaria» y «no ha existido intención compositiva al guna». En el presupuesto se cuantificaba que todas las demoliciones costarían 9.820 .000 pesetas y que la obra se cifraba en 44.863 .800 pesetas.

Durante estos meses, hay que destacar la publicación de diferentes artículos firmados por Luciano Tejedor respecto a la conservación del patrimonio de M elilla, y en este sentido escribía el 13 de febrero de 1981 un artículo denominado «Es necesario el esfuerzo y preocupación de todos. Hay que salvar el patrimonio arquitectónico de nuestra ciudad», ${ }^{5}$ donde con val entía se lamentaba amargamente de lo que parecía resultar obvio: la destrucción anunciada del Cine M onumental.

Benavides por el Ministerio de 0 bras Públicas (MO PU), Luciano Tejedor Mata y Manuel Corbí Ávila. Como invitados asistieron el alcal de Rafael Ginel Cañamaque y el del egado del M O PU Laureano Folgar Villasenín.

4. El D elegado Provincial de Cultura y presidente de la Comisión, Enrique lbáñez N ar váez, daba cuenta a la prensa de la celebración de esta Comisión y que se había aprobado el anteproyecto de reforma «esta comisión no entra en los detalles para el aprovechamiento del interior del edificio y sí en las fachadas a las calles».

5. El Telegrama de M elilla, 13 de febrero de 1981. 
Mientras tanto el proceso continuaba sus pasos, y el 20 de febrero de 1981 se obtenía el visado del proyecto por el colegio oficial de arquitectos de Andalucía O riental, por lo que el día 21 el propietario solicitaba la preceptiva licencia de obras al Ayuntamiento. Y el 13 de marzo, la Comisión Permanente de este organismo, de acuerdo al informe de la Comisión de Arquitectura y U rbanismo aprobaba el proyecto básico, recomendando al gunas prescripciones secundarias relativas a la decoración lateral y concediendo la licencia municipal de obras, aunque exigía un proyecto de ejecución y daba cuenta de todo a la comisión de Patrimonio.

Las posturas no debían estar demasiado claras por entonces, porque esta Comisión de Patrimonio fue convocada el 27 de marzo de una forma que podríamos denominar cuanto menos como «extraordinaria». La reunión se celebró en la sede de la Delegación del Gobierno y no en la habitual de Cultura y estuvo presidida por el Delegado del Gobierno, a la vez Comandante General de M elilla, José María Bourgón Lopez-D óriga. ${ }^{6}$ En esta comisión fue aprobada por unanimidad de los que tenían derecho a voto la reforma firmada por el arquitecto J osé M aría Bourgón de Izarra, al haberse presentado una fachada lateral alternativa y producirse al gunos cambios ornamentales basados en las columnas-pilastras de fachada. El mismo diario El Telegrama de Melilla daba cuenta el día 29 de la reunión y de la aprobación del proyecto, «por cuanto recoge la esencia de la arquitectura modernista tan estimable en la M elilla de principios de siglo y cuyos val ores arquitectónicos y artísticos hacen de nuestra ciudad un verdadero museo modernista».

Hay que señalar que el grado de conocimiento por entonces del patrimonio arquitectónico melillense era prácticamente nulo, no existían monografías ni trabajos científicos que hubieran desvelado la importancia de la ciudad y para la casi totalidad de la sociedad melillense, los términos M odernismo y Art D éco eran realmente desconocidos. Sólo se apreciaron en estos momentos la sensibilidad de pequeños pero muy ac-

6. Según reflejaba El Telegrama de Melilla, a esta comisión extraordinaria asistieron Enrique Ibáñez como Delegado del Ministerio, Francisco M ir Berlanga como cronista de la ciudad, Joaquín Rodríguez Puget representante de servicios técnicos del Ayuntamiento, José LuisAlcaláVargas representante de centros culturales, Emilio Boj González representante del Ayuntamiento, y como invitados el alcalde Rafael Ginel Cañamaque y LucianoTejedor M ata por el ministerio de Cultura, siendo el secretario de la comisión Manuel Corbí Ávila. Señalar que Luciano Tejedor había pasado de figurar como miembro de la comisión (6 de octubre de 1980) a invitado (desde 19 de febrero de 1981), por lo que había perdido el derecho a voto. 
tivos grupos concienciados en la necesidad de defender el patrimonio, pero muy fragmentados en cuanto a sus objetivos. Ya hemos señalado las Ilamadas a la conciencia ciudadana que se desprenden de los artículos de Luciano Tejedor, pero también hay que sumar las actuaciones que se llevaron a cabo desde la recién creadaA sociación de Estudios M elillenses y desde algunos medios de comunicación, caso del programaTertulia Cultural, emitido por Radio M elilla de la cadena SER. ${ }^{7}$

En abril de 1981 la situación parecía dar un brusco cambio, y se inicia un proceso administrativo promovido por un melillense, Julio Bassets Ruttlant, que intentó activar el expediente para incoar el cine M onumental y evitar con ello una destrucción que ya parecía inevitable. El 21 de abril J avier Tussell, Director General de BellasArtes, Archivos y Bibliotecas del M inisterio de Cultura daba por incoado el expediente al Cine M onumental para declararlo monumento histórico artístico y daba cuenta al Ayuntamiento que las obras que se realizaran en él deberían contar con la aprobación previa de esa Dirección General. Con esta actuación, se inicia un dilatado y a veces confuso expediente, sal picado de acusaciones, contradicciones y falta de sensibilidad.

M ayo puede ser considerado el mes en el que la noticia salta a la opinión pública y se agudiza una polémica que se diver sifica de acuerdo a los actores implicados. El asunto aparece el 8 de mayo de 1981 en las páginas de El Telegrama de M elilla ${ }^{8}$ y este diario se hacía eco de la actuación de Javier Tussell. También se apuntaba que en una sesión plenaria del Ayuntamiento el concejal José Cortes había propuesto que se comprara el cine para ubicar en él una casa de la cultura, pero su propuesta no fue tenida en cuenta. La prensa escrita también recogía por entonces diferentes opiniones, que coincidían generalmente en destacar la conservación de la fachada principal del cine como un logro.

El día 10 se producen unas declaraciones del Delegado de Cultura ${ }^{9}$ y se referencia una reunión en laA sociación de Estudios M elillenses convocada por la vocalía de patrimonio que dirigía por entonces Jesús M iguel Sáez Cazorla con el fin de salvar el

7. Tertulia Cultural, programa emitido los lunes a las 18.30, contaba con la participación de Ángel Castro, Isabel Morán, Antonio Padilla, José Ramón Gonzál ez y Joaquín Díaz.

8. «El expediente de declaración de monumento histórico-artístico del cine M onumental, firmado el 21 de abril». El Telegrama de M elilla, 8 de mayo de 1981.

9. «Tras la incoación del expediente. Las obras de reforma del cine monumental podrían ser detenidas» El Telegrama de M elilla, 10 de mayo de 1981. Posteriormente, 23 de mayo, Enrique Ibánez publicaría otra entrevista en la que criticaba las actuaciones privadas que habían dado lugar a la incoación: «iporqué no se actuó en colaboración con los organismos locales y sí de forma secreta?». 
cine, planteando una recogida de firmas y proponiendo también que fuera dedicado a casa de la cultura. ${ }^{10}$

El 12 de mayo, el promotor de las obras, A shok J. Lalchandani publicaba una carta en El Telegrama de M elilla" defendiendo la propuesta de reforma y todas sus actuaciones. A puntaba que llevaba unos once meses haciendo gestiones (lo que nos remonta a junio de 1980) y que las obras aún no habían comenzado, desmintiendo todo lo que se había denunciado en la prensa. Por otra parte señal aba que cuando final izaran los trabajos cedería una amplia zona en una de las plantas al Ayuntamiento de M elilla para realizar la casa de la cultura, además de pensar que su proyecto generaría «cien puestos de trabajo». Al día siguiente se publicaba una carta de Julio Bassets, ${ }^{12}$ iniciador del expediente de incoación, donde criticaba a la Comisión de Patrimonio por haber permitido las obras. Finalmente el día 14 de mayo de 1981 un telegrama de la Dirección General de Cultura ordenaba la paralización de las obras de derribo del cine, al no estar autorizadas, pero cinco días más tarde se desdecía al comprobar que había recibido autorización previa de la Comisión de Patrimonio de M elilla.

La propiedad buscó entonces una forma efectista de convencer a las fuerzas vivas de M elilla de la bondad de las obras, y el 16 de mayo anunciaba la presentación de una maqueta del proyecto. ${ }^{13}$ Ese mismo día El Tel egrama de M elilla reproducía un artículo publicado en El Telegrama del Rif el día de la inauguración del cine, el 13 de marzo de 1932. El propio medio reconocía que las opiniones estaban muy divididas al respecto. ${ }^{14}$ La presentación de una maqueta de cómo quedaría el cine después de las obras a escala 1:75, fue un conseguido golpe de efecto (Fig. 22). La noche del 18 de mayo se mostraba

10. En relación a la campaña que se produjo en M elilla a favor de la sal vación del cine, caben destacar las actuaciones que se realizaron desde laA sociación de Estudios M elillenses. Uno de los capítulos más desconocidos de este proceso, es que su presidente, Francisco Saro Gandarillas, entonces comandante del Ejército, no pudo continuar ejerciendo la presidencia al no recibir la correspondiente autorización que debía conceder la Comandancia G eneral de M elilla. Dejaría su cargo el 17 de octubre de 1981.

11. «Se crearían mas de cien puestos de trabajo con la reforma del cine monumental». El Telegrama de Melilla, 12 de mayo de 1981.

12. «Si alguien insinúa que he obrado con intencionalidad, miente descaradamente». El Telegrama de Melilla, 13 de mayo de 1981.

13. «El próximo lunes será presentada la maqueta del proyecto de reforma del Cine Monumental», El Telegrama de M elilla, 16 de mayo de 1981.

14. Este día se publicaba una carta de Manuel Guirval Pérez solicitando que el cine monumental fuese convertido en casa de la cultura. 
la maqueta a los medios de comunicación, con la presencia de buena parte de las autoridades de la ciudad y en las noticias del día siguiente, se daba cuenta del acto y se daba a entender, muy discutiblemente, que todos los asistentes estaban de acuerdo con la reforma. En este contexto, se produjo una desafortunada declaración del Cronista 0 ficial de M elilla, Francisco Mir Berlanga que fue resaltada y ampliamente utilizada por aquellos que querían sacar adelante la destrucción del cine: «El cine M onumental, artísticamente es una birria e históricamente no tiene importancia». ${ }^{15} \mathrm{M}$ ir Berlanga era por entonces un respetado historiador, pero desconocía en absoluto cualquier extremo vinculado con la arquitectura del siglo XX en general y con el Art Déco en particular, por lo que su lapidaria frase no deja de ser un clarísimo ejemplo de la necesidad de que uno no debe opinar nunca sobre lo que no entiende. Sin embargo, su autoridad de criterio fue utilizada ampliamente para justificar la transformación, y en un artículo favorable a la transformación firmado por KAKE ${ }^{16}$ ya se evidencia cómo había calado esta presentación.

Las posturas se decantaban hacia la conservación total o hacia la transformación, porque en el fondo nunca se situó el debate en lo que era una obviedad, y es que realmente se trataba de una demolición encubierta y lo que se debía discutir era la desaparición de la parte más significativa del edificio. El 13 de mayo la asamblea de la Sociedad Cultural Amigos de la M úsica solicitaba la conservación y la creación de la casa de la cultura en el cine, mientras que A gustín Jérez, ${ }^{17}$ arremetía en un artículo irónico contra el supuesto valor del monumento y Andrés Hernández también se mostraba partidario de la transformación. ${ }^{18}$

Sin embargo otros artículos ya introducían elementos de análisis de más interés sobre el cine y su valor estético, como el publicado por M iguel Ángel Suárez Garmendia, ${ }^{19}$ que efectuó una aproximación estilística al cine en sí, y también unas

15. «El cine M onumental, artísticamente es una birria e históricamente no tiene importancia». El Telegrama de Melilla, 19 de mayo de 1981.

16. KAKE. «Esto va a quedar aśi», M elilla, mayo de 1981. Se mostraba una foto de la maqueta.

17. El ciudadano Agustinof (Agustín Jerez Samper). «A propósito del cine Monumental. Galgos o Podencos». El Telegrama de Melilla, 20 de mayo de 1981.

18. Hernández, Andrés. «Entrevista con el cine M onumental» El Telegrama de Melilla 15 de julio de 1981, p. 7.

19. Suárez Garmendia, Miguel Ángel. «Sobre la metamorfosis del Cine M onumental y otros anexos». El Telegrama de M elilla. 26 de mayo de 1981. 
brevísimas y aceradas notas sobre la sociedad melillense repletas de contenido crítico, subrayando las «especial es características de su burguesía... la lumpen-aristocracia melillera, su mesocracia y su burguesía de tenderos» que «sueñan por celebrar se en la escenografía de los palcos del M onumental... ». Garmendia presentía la posibilidad del inicio de un desarrollismo incontrolado que liquidara la herencia arquitectónica de M elilla y alertaba sobre ello.

El debate fue subiendo de nivel científico, gracias a la aportación de profesores e investigadores del resto de España, y en junio encontramos la participación de arquitectos como Alejandro García Molinazo y sobre todo del que fuera uno de los descubridores del modernismo melillense, el arquitecto y ensayista catalán Salvador Tarragó Cid, ${ }^{21}$ que aportó al respecto nuevas y enriquecedoras visiones: así se recuperó por vez primera el término Art Déco aplicado a la arquitectura melillense, e incluso se subrayaba por entonces cierto ambiente futurista en los interiores, enriqueciéndose con ello su panorama estilístico y aplicando una terminología desconocida para el gran público melillense. En esta misma línea se produjeron importantes adhesiones para la conservación del Cine, como la firmada unánimemente por el Departamento de Historia del Arte de la U niversidad de M álagaz22 que defendía el carácter Art Déco del edificio 0 de José Luis $N$ avar ro Lara, ${ }^{23}$ graduado en artes aplicadas que añadía un análisis sobre su decoración introduciendo al gunas notas sobre moldurajes, repeticiones formales y simetrías.

Sin embargo, las obras de demolición continuaban su proceso de una forma imparable, a pesar de las voces al tamente autorizadas y cual ificadas que cuestionaban el proyecto. El día 1 de septiembre de 1981 comenzaban oficialmente las obras, aunque ya anteriormente se habían venido real izando trabajos interiores (Figs. 23 a 27). Desde entonces la polémica continúa de una forma mas áspera, involucrándose en ella el propio

20. García M olina, Alejandro. 06-06-1981. «Cine M onumental: una propuesta conciliadora» El Telegrama de M elilla, 6 de junio de 1981. Este arquitecto de Málaga proponía la conservación de la fachada lateral y del hall.

21. Tarrago Cid, Sal vador. «Cine M onumental: Edificio Histórico-Artístico de interés provincial». El Telegrama de Melilla. 9 de junio de 1981.

22. Departamento de H istoria del Arte de la U niversidad de Málaga, (D irectora D ña. Rosario Camacho M artínez). «El Departamento de Historia del Arte de la Universidad de Málaga en defensa del Cine Monumental de Melilla». El Telegrama de M elilla. 23 de junio de 1981.

23. Navarro Lara, José Luis. «Análisis de la decoración interior del Monumental Cinema Sport. El ayer de un tema polémico hoy». El Telegrama de M elilla. 12 de julio de 1981. 
PSOE de M elilla, que el 13 de octubre de 1981 pedía al Colegio deArquitectos la paralización de las obras por incumplir el Plan general. Ese mismo día el citado Colegio se reunió y de forma impactante anulaba el visado concedido por cuestiones urbanísticas y comunicaba su decisión al Ayuntamiento, al Delegado del Gobierno y a la propia Comisión de Patrimonio.

Como era de esperar, la prensa local recogió esta decisión y el 22 de octubre ${ }^{24}$ se podía leer: «Denuncia contra la reforma del cine M onumental, por infracción urbanística grave». U na serie de cartas e informes cruzados entre el Colegio de Arquitectos y el Ayuntamiento no consiguieron otra cosa que enrarecer más la situación, mientras las obras de demolición llevadas a cabo por la empresa Cubiertas y MZOV seguían su curso. Esta fase finaliza con un informe realizado por el arquitecto municipal de M elilla25 en el que reafirmaba la legalidad de las actuaciones anteriores y hacía constar que en el Ayuntamiento no constaba real mente proyecto de demolición al guno (ii i). Por su parte, esta misma institución llegó a cuestionar las actuaciones del Colegio de Arquitectos, mientras que las posibilidades de salvar el edificio desaparecían debido a los intereses y las voluntades que ya han aparecido nítidamente recogidas en estas notas, y enterradas por los propios mecanismos de una burocracia barroca y delirante.

Finalmente, el cine fue totalmente destruido en su interior y en su fachada lateral, manteniéndose la fachada principal como testigo de lo que una vez fue. Posteriormente la historia del edificio ha corrido por derroteros muy diversos, ${ }^{26}$ pero la memoria colectiva de M elilla lo asume actual mente como una lamentable pérdida.

El año 1985 representó un momento importante para el conocimiento del pa trimonio de la ciudad al celebrarse en ella dos eventos muy significativos. Del 18 al 22 de marzo, la Dirección Provincial del M inisterio de Cultura celebró un congreso sobre arquitectura modernista polarizando el interés de diferentes investigadores nacionales sobre M elilla. Para el citado Congreso, la profesora de la U niversidad de Málaga Rosa-

24. «Denuncia contrala reforma del cine monumental, por infracción urbanística grave». El Telegrama de M elilla, 22 de octubre de 1981.

25. «Cine M onumental: Finalizado el informe del arquitecto municipal (Fernando Moreno Jurado)» El Telegrama de M elilla, 27 de noviembre de 1981.

26. Incluso cuando nadie pensaba que se podía realmente empeorar lo proyectado, la real idad vino a quitar la razón y en junio de 1985 se solicitaba permiso a la Comisión de Patrimonio para instalar un bingo en el interior del edificio, lo que exigía una escalera de emergencia que generó nuevos y far ragosos expedientes para mitigar su impacto. Finalmente se materializaba, eso sí, de forma insultante, la vieja idea de crear en su interior una casa de la cultura. 
rio Camacho Martínez ${ }^{27}$ presentó un trabajo centrado sobre la importancia del Art Déco en esta ciudad, analizando cómo la construcción del Cine M onumental por el arquitecto Lorenzo Ros Costa influyó en muchas obras de Enrique N ieto y N ieto. Sobre este mismo temaArt Déco volvería Rosario Camacho con posterioridad en otra de sus publicaciones, ${ }^{28}$ aportando nuevos datos sobre la obra de Lorenzo Ros Costa y su vinculación con la ciudad norteafricana (Figs. 28 y 29).

El mismo año se celebraría también el Simposium Nacional del Comité Español de Historia del Arte, con nuevas aportaciones de varios investigadores al respecto. El profesor Javier Pérez Rojas, ${ }^{29}$ presentó una conferencia sobre el ya desaparecidoTeatro Cine Monumental, viendo en él una de las expresiones arquitectónicas más cosmopolitas de toda España en esta corriente. Estas impresiones fueron ampliadas posteriormente (1991) en el fundamental libro que Pérez Rojas escribió sobre Art D éco, ${ }^{30}$ donde explicaba el carácter de modernidad que revistió este estilo en su época, sobre todo en lugares al ejados de las obras de vanguardia (Figs. 30 a 34).

Estos congresos fueron el preámbulo y el remate de los trabajos de catalogación del patrimonio llevados a cabo por la Comisión de Patrimonio de M elilla, que condujeron finalmente a la declaración de una parte de la ciudad como Bien de Interés Cultural con la categoría de conjunto histórico, mediante Real decreto 2751-1986 de 5 de diciembre. Aunque esta declaración llegaba tarde para un edificio emblemático como el Cine Monumental, permitió la base legal para salvar de la piqueta otros muchos y ha sido el mecanismo de protección del Patrimonio vigente hasta nuestros días.

La destrucción del Cine M onumental fue un hito importante en su momento, y marcó una época y a una parte de la sociedad de M elilla que empezó a comprender, en ese periodo de los inicios de la Democracia, que no podía evolucionar sin defender sus señas de identidad, plasmadas entre otros aspectos, en su arquitectura modernista y art déco.

27. Camacho M artínez, Rosario. «Las sugestiones del Art Déco en la arquitectura de MeliIla». Boletín deArte, n. ํ 7. Málaga: Universidad, 1986; pp. 155 a 167.

28. Camacho Martínez, Rosario. «El arquitecto Lorenzo Ros y Costa y la difusión del Art Déco en Melilla». En: Arquitectura y ciudad. Seminario cel ebrado en Melilla, los días 12,13 y 14 de diciembre de 1989. Madrid: ICRBC., 1992; pp. 57 a 66.

29. Pérez Rojas, Javier. «Sobre la arquitectura del cine en España. El cine Monumental de M elilla». El barco como metáfora visual y vehículo de transmisión de formas. Actas del simposio nacional de Historia del Arte, CEH A. Málaga-M elilla, 1985. Málaga: varios editores, 1987; pp. 275 a 290.

30. Pérez Rojas, Javier. Art D éco en España. Madrid: Cátedra, 1990; p. 645. Existen en esta obra 19 referencias sobre Melilla y dos fotografías. 


\section{LA DESTRUCTION DU CINÉMA MONUMENTAL ET LA DÉFENSE DU PATRIMOINEART DÉCO}

Antonio Bravo N ieto UNED - U niversité de Málaga, Espagne

Le cinéma M onumental, construit sur le projet de l'architecte de Carthagène Lorenzo Ros, fut inauguré en mars 1932 (Figs. 1, 2 et 3). La presse locale de M elilla se fit l'écho en ce temps-là de la nouvelle dans plusieurs de ses éditions. El telegrama del Rif publiait le 13 mars 1932 un article intitulé «En la presente semana se inaugura el monumental» («cette même semaine on inaugure le monumental») et le 20 mars un autre travail annonçait: «Se ha inaugurado el M onumental Cinema» («le Cinéma M onumental a été inauguré»).

Peu de personnes pouvaient alors imaginer que le fastueux bâtiment qui, à ce moment-là, éblouissait et laissait perplexes les habitants de M elilla à cause de son aspect monumental et de sa nouveauté, ne fêterait même pas son demi siècle (Figs. 5 à 20). U ne intervention immobiliaire réalisée au début des années quatre-vingt mettrait fin à l'oeuvre principale de l'Art Déco de Melilla et de l'une des plus importantes construites dans ce style dans le territoire espagnol. Au cours de cette petite étude, nous allons nous centrer sur la description d'un aspect très peu connu de ce bâtiment, sur le récit du processus qui détermina sa destruction, et qui se déroule fondamentalement entre les années 1980 et 1981 (Fig. 21).

La crise caractéristique subie par le cinéma depuis les années soixantedix du XX $X^{\mathrm{e}}$ siècle, ébranla les possibilités commerciales du Cinéma M onumental et la famille Liarte, propriétaire de l'immeuble, réalisa sa vente après une longue période de décadence. La protection du patrimoine architectural à M elilla à la fin des années soixante-dix se trouvait encore dans une phase très embryonnaire et en ce temps-là les fondements de ce que serait le catalogage définitif des nouveaux quartiers style Art N ouveau de la ville. Dans le même ordre d'idées, le 9 juillet 1979 La Commission du Patrimoine de Melilla et le Représentant Provincial du 
M inistère de la Culture essayaient de réaliser un premier inventaire des immeubles à protéger. ${ }^{1}$

En ce qui concerne la politique, la ville était gouvernée par le parti U nión del Centro D emocrático auquel appartenait Rafael Ginel Cañamaque élu maire le 19 avril 1979. Le 22 juin de cette même année, José M aría Bourgón López Dóriga avait été nommé Commandant Général de M elilla et Préfet. ${ }^{2}$

Les travaux de la Commisssion du Patrimoine récemment créée avaient pour but de constituer des dossiers pour déclarer d'intérêt général les bâtiments les plus représentatifs de M elilla. Le 11 février, les premières démarches commencèrent et le 26 de ce même mois le M inistère de la Culture sollicitait de son représentant une liste d'éventuels bâtiments qui pour raient être déclarés monuments historiques et artistiques. En réponse à cette iniciative, la commission remit le 11 mars au Directeur Général une liste de 25 bâtiments ayant un intérêt manifeste qui permettait d'entreprendre leur protection; parmi eux se trouvait le Cinéma M onumental.

Cependant, la vente du Cinéma M onumental était déjà un état de fait qui se négociait à ce moment-là, et en juillet 1980, son nouveau propriétaire, Ashok J hamandas Lalchandani sollicitait les services de l'architecte José M aría Bourgón de Izarra pour transformer l'ancien cinéma en un immeuble de locaux commerciaux. Le 4 août 1980, le propriétaire remettait au Président de la Commission du Patrimoine Historique et Artistique de M elilla un écrit auquel était jointe l'étude préal able de la transformation

1. Compte-rendu n. -5 du 6 octobre 1980. 0 nt assisté à cette commission le président et le Représentant du M inistère de la Culture Enrique Ibañez N arváez, Francisco Mir Berlanga en tant que représentant des corporations locales, Joaquín Rodríguez Puget en tant que représentant des Services Techniques Alfonso Álvarez Benavides pour le M inistère desTravaux Publiques (M O PU ), Luciano Tejedor Mata et $M$ anuel Corbí Ávila. O nt asisté en tant qu'invités le maire R afael Ginel Cañamaque et le représentant du M O PU Laureano Folgar Villasenín.

En décembre 1978 la réalisation d'un inventaire architectural de M elilla fut adjugée. Les tra vaux de classement des bâtiments furent effectués en mai 1979 et l'inventaire fut remis en janvier 1980. Cet inventaire comprenait en réalité 152 fiches, 68 photographies et un mémoire de 7 pages. Á la même époque, en décembre 1979, Luciano Tejedor Mata réal isait quelques-uns des premiers inventaires connus des bâtiments les plus impor tants de M elilla, qu'il catal oguait comme appartenant àl'Art N ouveau, à l'Historicisme et au Rationalisme.

2. Pendant cette période, le Commandant Général de la ville exerçait en même temps la charge de Préfet, et en temps que tel, supérieur du représentant du Ministère de la Culture à M elilla. José María Bourgón y López Dóriga fut nommé le 22 juin 1979 et il finit son mandat le 14 août 1981. Précédemment il avait exercé la fonction de Directeur du CESID. 
du cinéma M onumental. L'idée première était d'approuver cette étude, pour développer a posteriori le projet et obtenir le permis de construire. D'autre part, la propriété envoyait à l'H ôtel deVille de M elilla les mêmes documents, avec les plans de la rénovation. L'H ôtel deVille répondait le 1 octobre tout en signalant que les Services d'Architecture considéraient que la modification était correcte et qu'elle respectait les normes du plan général d'urbanisme.

Pour sa part, la Commission du Patrimoine se réunissait le 6 octobre de cette même année 1980 dans une séance qui se révèlerait capitale en ce qui concerne le sort du M onumental. D'un côté, on étudia le début des formalités de d' ouverture des dossiers de 10 des 25 immeubles proposés, mais le sujet fondamental fut létude de l'avant-projet de rénovation pour son approbation ou sa dénégation. A près un long débat, les assistants approuvèrent I'avant-projet présenté, Luciano Tejedor M ata fut le seul membre de la Commission qui vota contre l'avant-projet car il considérait qu'il ne respectait ni les zones intérieures ni la façade latérale. À cette époque, la Commission veillait plus à conserver l'image externe qu'à préserver l'intérieur, c' est pour cela, en réal ité, que l'avenir du cinéma fut condamné à l'échec à partir de cet instant. Curieusement, dans la même réunion il fut décidé d' ouvrir le dossier de déclaration des dix immeubles les plus significatifs de la ville parmi lesquels se trouvait le ci-dessus cité M onumental.

En janvier 1981 l'architecte J osé M aría Bourgón de Izarra terminait le «Projet de rénovation du cinéma Monumental, pour le convertir en immeuble de bureaux et locaux commerciaux, situé dans les ruesA. de Mera et Ejército Español, à M elilla, pour D. Ashok Jhamandas Lalchandani». Le projet se composait d'une mémoire, d'un devis et de plans, numérotés du 1 au 7 qui correspondaient àl'état actuel et du 8 au 23 correspondants au projet initial. L'architecte qui justifiait le dit projet de rénovation, indiquait, en se référant aux travaux de 1932, que la façade latérale original avait été «soumise à un traitement secondaire» et «qu'il n'y avait eu aucune intention esthétique». Le devis spécifiait que toutes les démolitions coûteraient 9.820 .000 pesetas y que les travaux se chiffraient à 44.863 .800 pesetas.

Pendant ces mois-là, nous soulignerons la publication de divers articles signés par Luciano Tejedor en relation avec la conservation du patrimoine de M elilla, et dans ce sens, il écrivait le 13 février 1981 un article intitulé «L'effort et la préoccupation de tout le monde sont nécessaires. II faut sauver le patrimoine architectural de notre ville». ${ }^{3}$ Dans cet article, il se lamentait vaillamment et avec amertume de ce qui semblait être évident: la destruction annoncée du Cinéma M onumental.

3. El Telegrama de M elilla, 13 février 1981. 
Pendant ce temps le processus continuait son cours, et le 20 février 1981, I'O rdre des architectes de l'Andal ousie 0 rientale validait le projet, le propriétaire sollicitait al ors le permis de construire àl'H ôtel deVille. Et le 13 mars, la Commision Permanente de cet organisme, en accord avec le rapport de la Commission d'A rchitecture et d'U rbanisme autorisait le projet initial, tout en recommandant quelques directives secondaires en relation avec la décoration latérale, et accordait le permis de construire, quoiqu' elle exigeait un projet d'exécution et elle rendait compte de tout cela à la Commission du Patrimoine.

Les opinions, à cette époque, ne devaient pas être très claires, car cette Commission du Patrimoine fut convoquée le 27 mars d'une façon qu'on pourrait qualifier tout au moins «d'extraordinaire». La réunion s'est tenue au siège de la Préfecture et non à celui du Représentant du M inistère de la Culture comme il était habituel; elle fut présidée par le Préfet et Commandant Général de M elilla, José M aría Bourgón LópezDóriga. ${ }^{4}$ Dans cette commission, la restauration signée par l'architecte J osé M aría Bourgón de Izarra fut approuvée par unanimité par ceux qui avaient le droit de vote car elle incluait une façade latérale alter native et présentait certains changements ornamentaux basés sur les colonnes-pilastres de la façade. Le même journal EL Tel egrama de M elilla rendait compte de la réunion et de l' approbation du projet, «car il conserve l'essence de l'architectureA rt N ouveau si appréciée dans la M elilla du début du siècle et dont les valeurs architecturales et artistiques font de notre ville un véritable muséeA $\mathrm{rt}$ N ouveau».

II nous faut remarquer que le degré de connaissance du patrimoine architectural de Melilla était pratiquement nul à l'époque, il n'existait pas de monographies ni de travaux scientifiques qui auraient pu révéler l'importance de la ville, et pour la quasi totalité de la société de M elilla, les termesArt Nouveau et Art Déco étaient réellement méconnus. On put seulement percevoir en ces moments-là la sensibilité de petits groupes mais cependant très actifs qui étaient conscients de la nécessité de défendre le patrimoine, mais ils avaient des objectifs très fragmentés. N ous avons déjà signalé les ap-

4. Selon EI Telegrama de M elilla, ont assisté à cette commission extraordinaire Enrique Ibáñez en tant que représentant du M inistère, Francisco M ir Berlanga en tant que chroniqueur de la ville, Joaquín Rodríguez Puget représentant des services techniques de l'H ôtel deVille, José LuisA IcaláVargas représentant des centres culturels, Emilio Boj González représentant de l'Hôtel deVille, et comme invités le maire Rafael Ginel Cañamaque et Luciano Tejedor $M$ ata pour le M inistère de la Culture, et $M$ anuel Corbí Á vila qui remplissait la fonction de secrétaire de la commission. N ous signalerons que Luciano Tejedor ne figurait plus comme membre de la commission (6 octobre 1980) mais comme invité (depuis le 19 février 1981) raison pour laquelle il n'avait pas droit de vote. 
pels à la conscience citadine qui se découlent des articles de Luciano Tejedor, mais il faut aussi y ajouter les actions qui furent engagées par la nouvellement crééeA sociación de Estudios M elillenses et par certains médias, comme ce fut le cas du programmeTertulia Cultural de la station de radio SER. ${ }^{5}$

En avril 1981, la situation paraissait subir un brusque changement, et c' est un processus administratif qui commence, promu par un habitant de M elilla, Julio Bassets Ruttlant qui essaya d'accélérer le dossier pour protéger le C inéma M onumental et éviter par cela même une destruction qui semblait déjà inévitable. Le 21 avril Javier Tussell, Directeur Général des Beaux Arts, Archives et Bibliothèques du Ministère de la Culture considérait commencé le dossier du Cinéma M onumental pour le déclarer monument historique et artistique et communiquait àl'H ôtel deVille que les travaux qui s'y réal iseraient devraient obtenir l'autorisation préal able de cette Direction G énérale. À l'occasion de cette procédure, s'ouvre un dossier dilaté et parfois confus, parsemé d'accusations, de contradictions et de manque de sensibilité.

$M$ ai peut être considéré comme le mois où la nouvelle parvient jusqu'à l'opinion publique et où s'accentue une polémique qui variait selon les personnes impliquées. L'affaire apparaît le 8 mai 1981 dans les pages du Telegrama de M elilla ${ }^{6}$ et ce journal se faisait l'écho de l'intervention de Javier Tussell. O $n$ y signalait aussi que pendant une séance plénière de l'Hôtel deVille, le conseiller municipal José Cortés avait proposé l'achat du cinéma pour y installer une maison de la culture, mais sa proposition ne fut pas retenue. À l'époque la presse écrite recueillait aussi différentes opinions, qui coïncidaient généralement pour souligner la conservation de la façade principale du cinéma comme une réussite.

Le 10, le Délégué du M inistère de la Culture7 fit des déclarations et le même journal recueilli une réunion à l'A sociación de Estudios M elillenses convoquée par la

5. Tertulia Cultural, émmision diffusée le lundi à $18 \mathrm{~h} 30$, qui comptait avec la participation de Ángel Castro, Isabel M orán, Antonio Padilla, José Ramón González y Joaquín Díaz.

6. «Le dossier de déclaration de monument historique et artistique du Cinéma M onumental, signé le 21 avril». El Telegrama de M elilla, 8 mai 1981.

7. «Après I'ouverture du dossier. Les travaux de restauration du cinéma M onumental pourraient être paralysés» El Telegrama de M elilla, 10 mai 1981. Plus tard, le 23 mai, Enrique Ibáñez publierait une autre interview dans laquelle il critiquait les actions de particuliers qui avaient été à l'origine de I'ouverture du dossier: «pourquoi n'avait-on pas agi en coll aboration avec les organismes locaux au lieu de le faire secrètement». 
section du patrimoine que dirigeait al ors Jesús M iguel Sáez Cazorla afin de sauver le cinéma, en requérant des signatures et aussi en proposant qu' on le consacre a une maison de la culture. ${ }^{8}$

Le 12 mai, le promoteur des travaux, A shok J. Lalchandani publiait une lettre dans le Telegrama de M elilla ${ }^{9}$ dans laquelle il défendait la proposition de rénovation et toutes ses actions. II signalait qu'il y avait onze mois qu'il faisait des démarches (ce qui remonte à juin 1980) et que les travaux n'avaient pas encore commencé. II démentait tout ce qui avait été dénoncé dans la presse. D'autre part, il soulignait que lorsque les travaux seraient terminés, il cèderait à l'H ôtel deVille une vaste zone dans un des étages pour réaliser la maison de la culture; en outre il pensait que son projet pourrait créér «cent postes de travail». Le lendemain, Julio Bassets, ${ }^{10}$ promoteur de I'ouverture du dossier, publiait une lettre dans laquelle il critiquait la Commission du Patrimoine pour avoir permis les travaux. Enfin le 14 mai 1981 un télégramme de la Direction Générale du M inistère de la Culture ordonnait l'arrêt des travaux de démolition du cinéma, car ils n'étaient pas autorisés, mais cinq jours plus tard, elle revenait sur sa décision en constatant qu'il existait une autorisation préal able de la Commission du Patrimoine de M elilla.

Le propriétaire chercha alors un moyen impressionnant de convaincre les forces vives de M elilla de la bonté des travaux, et le 16 mai il annonçait la présentation d'une maquette du projet. " Le jour même El Telegrama de M elilla reproduisait un article publié dans le même journal (El Tel egrama del Rif) le jour de l'inauguration du cinéma, le 13 mars 1932. Le journal reconnaissait que les opinions à ce sujet étaient très divisées. ${ }^{12}$ La présentation d' une maquette exposant, à l'échelle de 1: 75, I'état final du ci-

8. En ce qui concerne lacampagne qui eut lieu àM elilla pour appuyer le sauvetage du cinéma, nous devons souligner les activités réal isées par l'Asociación de Estudios M elillenses. L'un des chapitres les moins connus de ce processus est que son président, Francisco Saro Gandarillas, commandant àce momentlà, ne put continuer à exercer sa charge car il n'avait pas reçu l'autorisation correspondante de la part du bureau du Commandant Général de Melilla. Sa démission se produit le 17-10-1981.

9. «Plus de cent postes de travail furent créés grâce à la restauration du cinéma M onumental». El Telegrama de M elilla, 12 mai 1981.

10. Si quelqu'un insinue que j'ai agi avec intentionnalité, il ment de manière honteuse». El Telegrama de Melilla, 13 mai 1981.

11. «lundi prochain, nous présenterons la maquette du projet de restauration du Cinema Monumental». El Telegrama de M elilla, 16 mai 1981.

12. Ce jour-là, une lettre de Manuel Guirval sollicitant la conversion du cinéma M onumental dans maison de la culture était publiée. 
néma provoqua un effet de surprise très réussi (Fig. 22). Dans la soirée du 18 mai, la maquette était montrée aux medias, avec la présence d'une bonne partie des autorités de la ville et, le lendemain, les informations rendaient compte de l'événement et laissaient entendre, de façon très discutable, que tous les assistants étaient d' accord avec la rénovation. Dans ce contexte, le C hroniqueur O fficiel de M elilla Francisco M ir Berlanga fit une déclaration malheureuse sur laquelle on mit l'accent et qui fut grandement utilisée par ceux qui voulaient que prospère la destruction du cinéma: «Le Cinéma M onumental, au niveau artistique est une horreur et historiquement il n'a aucune importance». ${ }^{13} M$ ir Berlanga était alors un historien respecté, mais il méconnaissait complètement quelconque notion liée à l' architecture du $X X{ }^{\circledR}$ siècle en général et de I'Art Déco en particulier, c'est pourquoi sa phrase lapidaire n'est qu' un exemple très clair de la nécessité de ne jamais donner son opinion sur ce qu' on ne connaît pas. Cependant, le crédit dont il jouissait fut largement utilisé pour justifier la transformation, et dans un article favorable à celle-ci signé par $K A K E,{ }^{14}$ on constatait déjà quelle influence avait eu cette présentation.

Les postures se penchaient vers la conservation totale ou vers la transformation, parce que dans le fond le débat ne s' est jamais situé sur ce qui était une évidence, car il s'agissait réellement d'une démolition dissimulée et ce que l'on devait discuter, c'était la disparition de la partie la plus significative de l'immeuble. Le 13 mai, l'assemblée de la Sociedad Cultural Amigos de la Música sollicitait la conservation et la création de la maison de la culture dans le cinéma, tandis que Agustín Jerez ${ }^{15}$ dans un article ironique s' en prenait à la valeur supposée du monument et Andrés Hernández se montrait également partisan de la transformation. ${ }^{16}$

Cependant, d'autres articles introduisaient déjà des éléments d' analyse plus intéressants sur le cinéma et sa val eur esthétique, comme celui publié par M iguel Ángel Suárez Garmendia, ${ }^{17}$ qui réal isa une approximation stylistique au cinéma même, et aussi

13. «El cinéma Monumental, est une horreur au niveau artistique et il n'a aucune importance historique», El Telegrama de M elilla, 19 mai 1981.

14. "Ça va être comme ça», M elilla, mai 1981. O n y montrait une photo de la maquette.

15. El ciudadano Agustinof (Agustín Jérez Samper). «A propósito del cine monumental. Gal gos o Podencos». El Telegrama de M elilla. 20 mai 1981. llet 1981, p. 7.

16. Hernández, Andrés. «Entrevista con el cine M onumental» El Telegrama de M elilla 15 jui-

17. Suárez Garmendia, Miguel Ángel. «Sobre la metamorfosis del Cine M onumental y otros anexos». El Telegrama de Melilla. 26 mai 1981. 
de brèves notes acerbes sur la société de M elilla, pleines de contenu critique, tout en soulignant « les caractéristiques spécial es de sa bougeoisie... le prolétariat aristocratique «melillero», son régime bougeois, et sa bourgeoisie de petits commerçants» qui «rêvent de se mettre eux-mêmes en scène dans les loges du M onumental. .» Garmendia présentait la possibilité du commencement d'un développement à outrance qui liquiderait I'héritage architectural de M elilla et il donnait l'alerte sur ce point.

Le niveau scientifique du débat s' éleva grâce à l'apport de professeurs et de chercheurs du reste de l'Espagne, et en juin nous découvrons la participation d'architectes tel que Alejandro García M olina ${ }^{18}$ et surtout de celui qui fut le découvreur de I'Art N ouveau de M elilla, I' architecte et essayiste catalan Salvador Tarragó Cid, ${ }^{19}$ qui apporta en ce domaine de nouvelles vues d'ensemble enrichissantes: c' est ainsi que pour la première foisle termeArt Déco fut récupéré pour l'appliquer àl'architecture de M elilla. Et à l'époque, on soulignait aussi une certaine ambiance futuriste dans les zones intérieures, ce qui enrichissait son panorama stylistique tout en appliquant une terminologie inconnue du grand public de M elilla. Sur ce même plan, d'importantes adhésions se produirent pour la conservation du Cinéma, telles que celle qui fut unanimement signée par le D épartement d'H istoire de l'Art de l'U niver sité de M alaga20 qui défendait le caractère Art Déco du bâtiment ou celle de José Luis N avarro Lara, ${ }^{21}$ diplomé en arts apliques, qui ajoutait une analyse sur sa décoration en introduisant quelques remarques sur les moulures, les répétitions de formes et les symétries.

Cependant, les travaux de démolition se poursuivaient d'un façon imparable, mal gré les opinions hautement autorisées et qual ifiées qui remettaient en question le projet. Le ler septembre 1981 les travaux commençaient officiellement, bien que des travaux avaient déjà été réalisés à l'intérieur (Figs. 23 à 27). Dès lors la polémique

18. García M olina, Alejandro, 06-06-1981. "Cine M onumental: una propuesta conciliadora» El Telegrama de M elilla 6 juin 1981. Cet architecte de M al aga proposait la conservation de la façade latérale et du hall.

19. Tarragó Cid, Sal vador. «Cine M onumental: Edificio Histórico-Artístico de interés provincial». El Telegrama de Melilla. 9 juin 1981.

20. Département d'Histoire de l'Art de M alaga. (Directrice M me. Rosario Camacho M artínez). «El Departamento de Historia del Arte de la U niversidad de Málaga en defensa del Cine M onumental de Melilla». El Telegrama de M elilla. 23 juin 1981.

21. Nanarro Lara, José Luis. «Análisis de la decoración interior del Monumental Cinema Sport. El ayer de un tema polémico hoy». El Telegrama de M elilla. 12 juillet 1981. 
continue d'une façon plus âpre, et même le PSO E de M elilla s' impliqua le 13 octobre 1981 en demandant àl'O rdre desArchitectes de paralyser les travaux pour ne pas avoir respecté le Plan Général d'U rbanisme. Ce même jour, I'O rdre desArchitectes se réunit et il annulait de manière saisissante l'autorisation accordée pour des raisons urbanistiques et il communiquait sa décision à l'H ôtel deVille, au Préfet et à la Commission du Patrimoine elle-même.

Comme il fallait s'y attendre, la presse locale enregistra cette décision et le 22 octobre 22 on pouvait lire: «D enuncia contra la reforma del cine M onumental, por infracción urbanística grave». U ne série de lettres et de rapports échangés entre l'O rdre des Architectes et la Mairie n'obtinrent d'autre résultat que celui de raréfier encore plus la situation, tandis que les travaux de démolition entrepris par l'entreprise Cubiertas y M ZOV suivaient leur cours. Cette phase se termine avec un rapport réal isé par l'architecte municipal de M elilla23 dans lequel il réaffirmait la légal ité des actions antérieures et où il faisait observer qu'à la Mairie il n'existait réellement aucun projet de démolition (i¿?!). De son côté, cette même institution en arriva à remettre en question les actions entreprises par l'O rdre desArchitectes, tandis que les possibilités de sauver le bâtiment disparaissaient à cause des intérêts et des désirs qui étaient déjà apparus clairement consignés dans ces notes, et enterrés par les mécanismes mêmes d'une bureaucratie barroque et delirante.

Final ement, le cinéma fut totalement détruit à l'intérieur ainsi que sa façade latérale. Seule la façade principale fut conservée en témoignage de ce qu' un jour il fut. Plus tard, I' histoire du bâtiment a suivi des voies très diver ses, ${ }^{24}$ mais la mémoire collective de M elilla l'assume actuellement comme une perte lamentable.

L'année 1985 représenta un moment important pour la connaissance du patrimoine de la ville car on y célébra deux événements très significatifs. Du 18 au 22

22. «D enuncia contra la reforma del cine monumental, por infracción urbanística grave». El Telegrama de M elilla. 22 octobre 1981.

23. «Cine M onumental: Finalizado el informe del arquitecto municipal (Fernando M oreno Jurado)». El Telegrama de M elilla, 27 novembre 1981.

24. M ême quand personne ne pensait que ce qui avait été projeté pouvait réellement empirer, la réalité nous démontra que c'était possible et en juin 1985 une autorisation était demandée à la Comisión du Patrimoine pour installer une salle de bingo à l'intérieur du bâtiment, ce qui rendait nécessaire la construction d' un escalier de secours qui provoqua de nouveaux dossiers confus et embrouillés pour réduire son impact. Finalement, la vieille idée de créer à l'intérieur une maison de la culture se matérialisait, mais certes de manière humiliante. 
mars, le Représentant du M inistère de la Culture organisa un congrès sur l' architecture Art N ouveau qui éveilla l'intérêt pour M elilla de différents chercheurs nationaux. La professeure de l'U niver sité de M alaga Rosario Camacho M artínez ${ }^{25}$ présenta à ce congrès un travail axé sur l'importance de l'Art D éco dans cette ville où elle analysait de quelle façon la construction du Cinéma M onumental par l'architecte Lorenzo Ros Costa eut de l'influence sur de nombreuses constructions réal isées par Enrique Nieto y N ieto. Plus tard, Rosario Camacho reprendrait ce même sujet Art Déco dans une autre de ses publications ${ }^{26}$ et apporterait de nouvelles données sur I' architecture de Lorenzo Ros Costa et ses liens avec la ville nord-africaine (Figs. 28 et 29).

Cette même année, le Symposium N ational du Comité Espagnol d'Histoire de l'Art se célébra et apporta de nouvelles contributions de plusieurs chercheurs dans ce domaine. Le professeur Javier Pérez Rojas²7 donna une conférence sur leThéâtre Cinéma M onumental, alors disparu, où il exposait qu'il le considérait comme l'une des expressions architecturales les plus cosmopolites de toute l'Espagne en ce qui concerne I'architecture Art Déco. Ces impressions furent ensuite développées (1991) dans le livre fondamental que Pérez Rojas écrivit sur l'Art Déco ${ }^{28}$ où il expliquait le caractère de modernité qui caracterisa ce style à son époque, surtout dans des endroits éloignés des oeuvres d' avant-garde (Figs. 30 à 34).

Ces congrès furent le préambule et le couronnement des travaux de classement du patrimoine menés à bien par la Commission du Patrimoine de M elilla, qui conduisirent finalment à la declaration d'une partie de la ville comme Bien d'Intérêt Culturel avec la catégorie d'ensemble historique, par le Décret Royal 2751-1986 du 5 décembre. Bien que cette déclaration soit ar rivée trop tard en ce qui concerne un bâtiment emblématique tel que le Cinéma M onumental, elle permit l'implantation des

25. Camacho Martínez, Rosario, «Las sugestiones del Art D éco en la arquitectura de MeliIla». Boletín de Arte, n. - 7. Málaga: U niversidad, 1986; pp. 155 à 167.

26. Camacho Martínez, Rosario. «El arquitecto Lorenzo Ros y Costa y la difusión del art déco en M elilla». Dans: Arquitectura y ciudad. Seminario celebrado en Melilla, los días 12,13 y 14 de diciembre de 1989. Madrid: ICRBC, 1992; pp. 57 à 66.

27. Pérez Rojas, Javier. «Sobre la arquitectura del cine en España. El cine Monumental de M elilla». El barco como metáfora vi sual y vehículo de transmisión de formas. Actas del simposio nacional de Historia del Arte, CEH A. M álaga-M elilla, 1985. Málaga: varios editores, 1987; pp. 275 à 290.

28. Pérez Rojas, Javier. Art Déco en España. M adrid: Cátedra, 1990; p. 645. Dans cet ouvrage, on peut trouver 19 références sur M elilla et deux photographies. 
fondements légaux pour sauvegarder beaucoup d'autres et elle s'est convertie en mécanisme de protection du Patrimoine actuel jusqu'à nos jours.

La destruction du Cinéma Monumental fut un événement marquant à son époque, et elle fut aussi le reflet d'une époque et d'une partie de la société de M elilla qui commença à comprendre, au moment où la Democratie faisait ses premiers pas, qu' elle ne pouvait évoluer si elle ne défendait pas sa propre identité, représentée, entre autres aspects, dans son architecture Art N ouveau et Art D éco. 



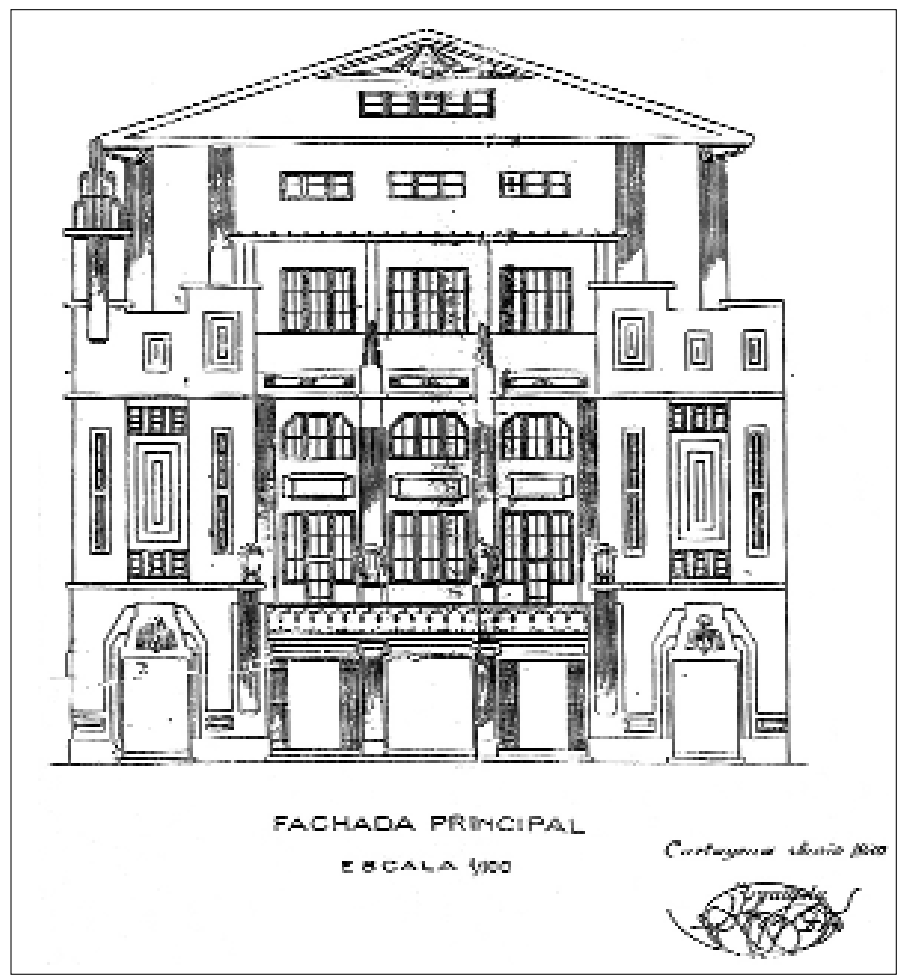

Fig. 1. Fachada del

Monumental Cinema,

proyecto de junio de 1930.

Fotografía del Archivo Central de M elilla (desde ahora ACM).

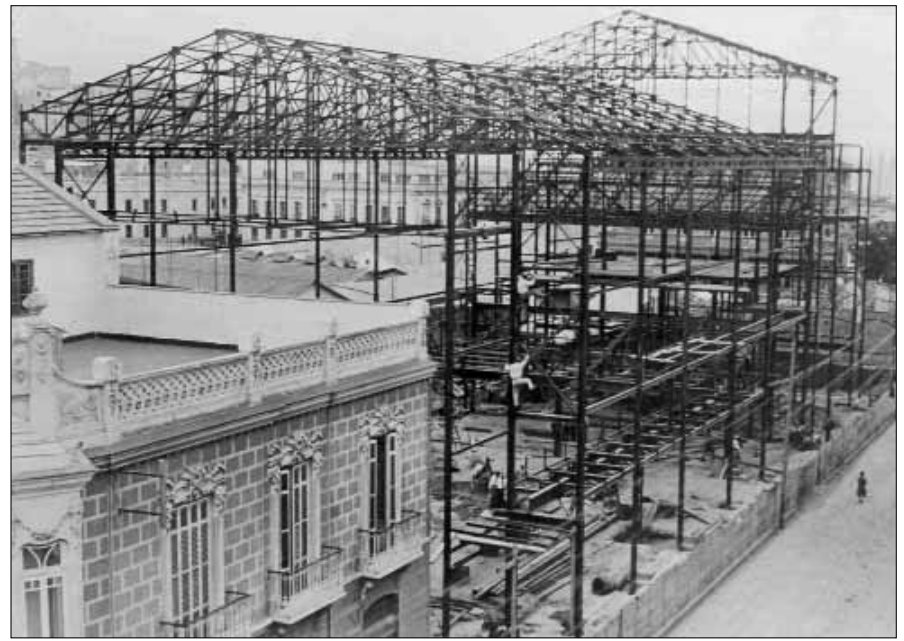

Fig. 2. Estructura metálica del edificio durante su construcción, 1930-1931 (ACM). 
Fig. 3. Vista frontal de la estructura metálica del cine, 1930-1931 (ACM).

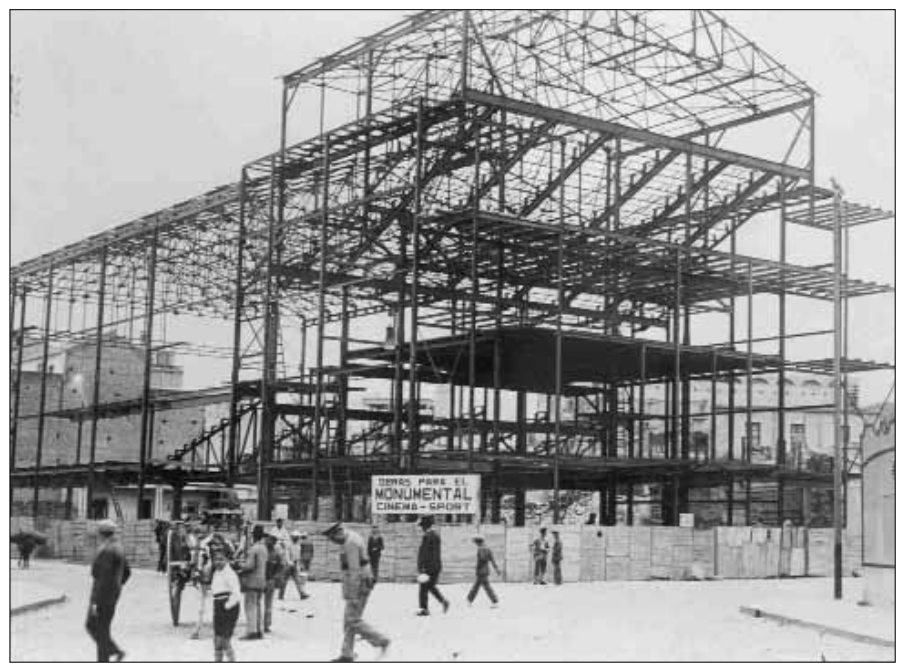

Fig. 4. El M onumental Cinema, 1932 (ACM).

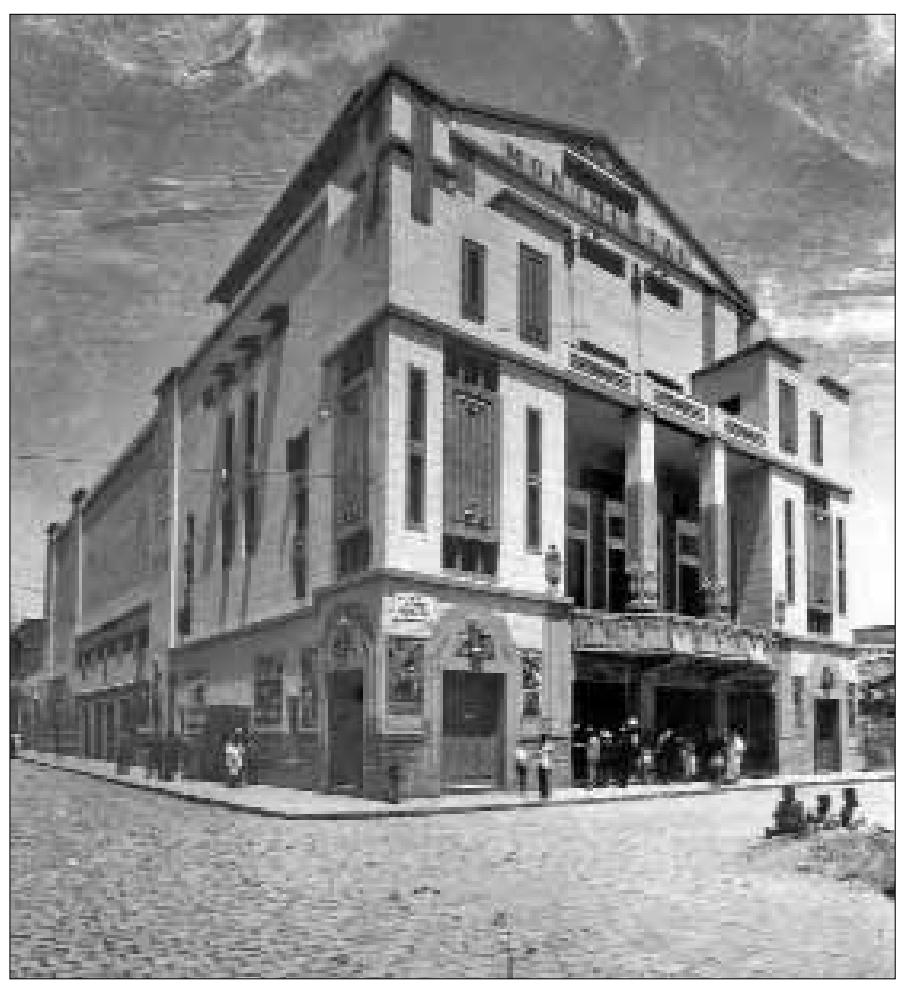




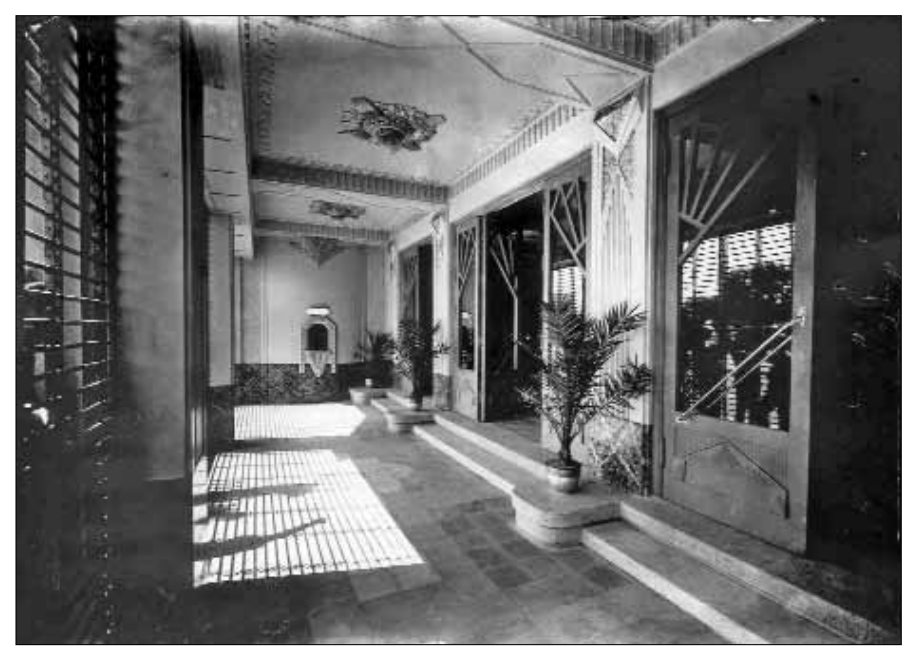

Fig. 5. Vistas de la zona de taquillas y puertas de entrada, 1932 (ACM).

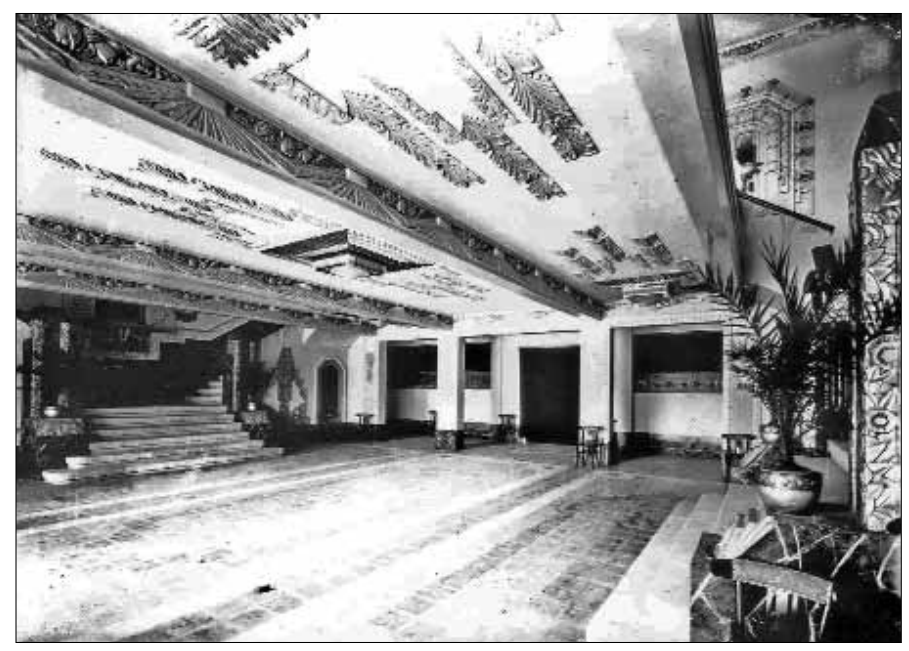

Fig. 6. Vista del gran hall de entrada, con acceso al patio de butacas, 1932 (ACM).

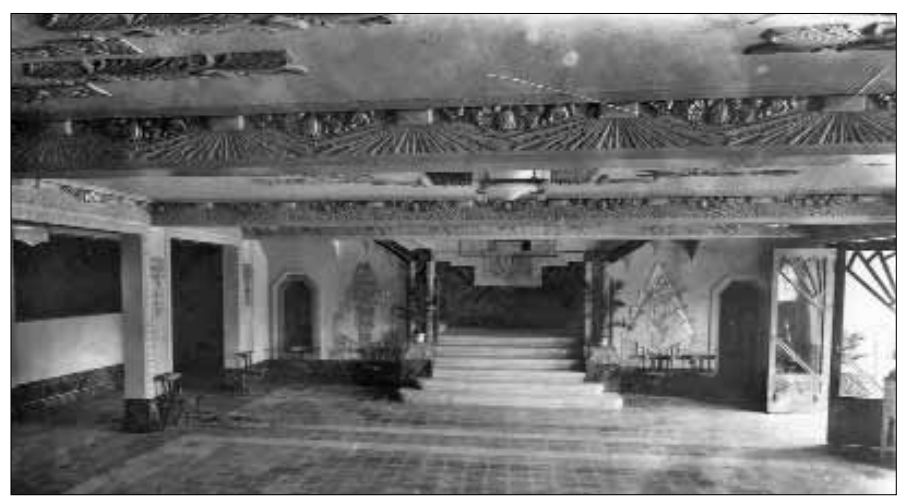

Fig. 7. Vista frontal de una de las escal eras de acceso a la planta principal, 1932 (ACM). 
Fig. 8. Detalle de una de las escaleras, 1932 (ACM).

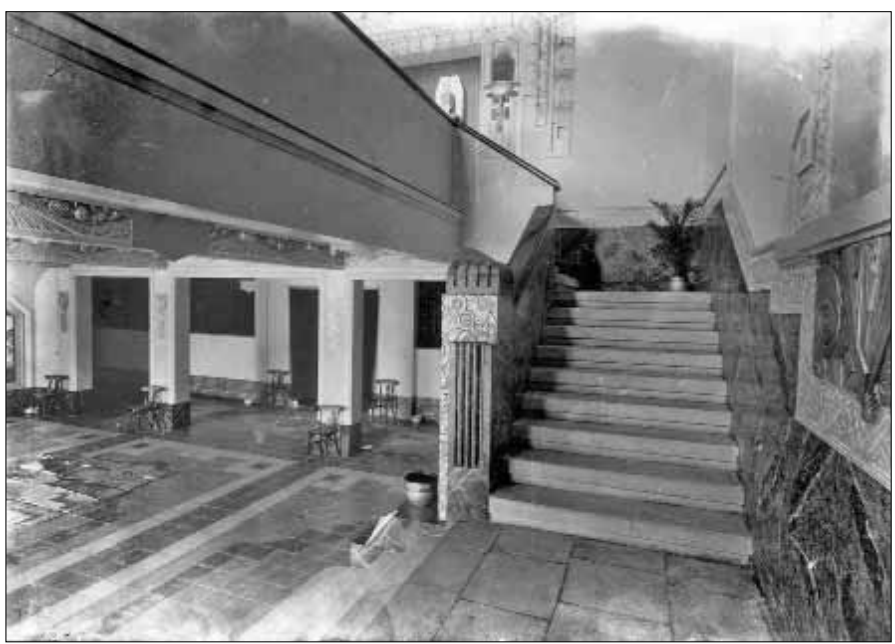

Fig. 9. Vista de la planta principal y ambigú, 1932 (ACM).

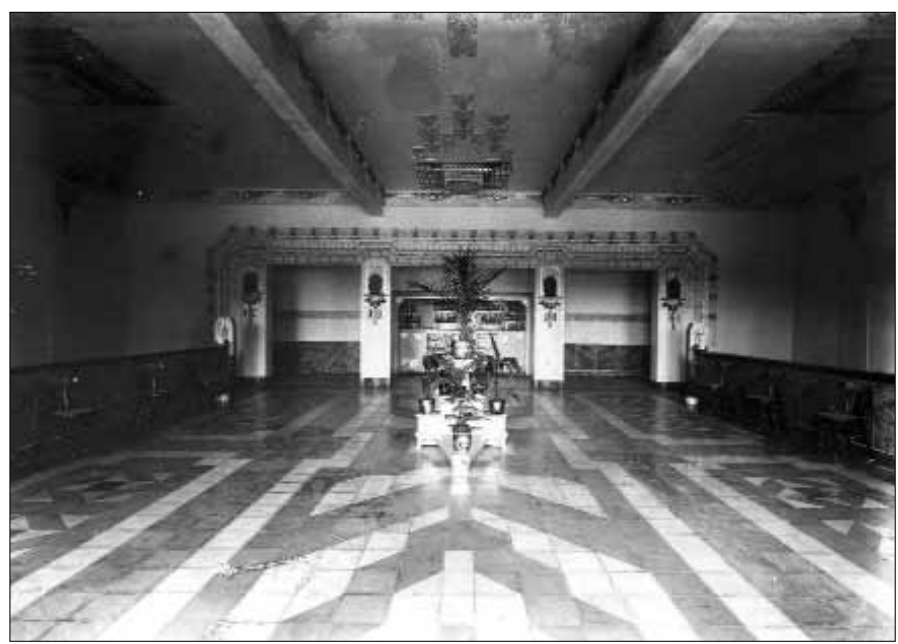

Fig. 10. Detalle de la planta principal, 1932 (ACM).

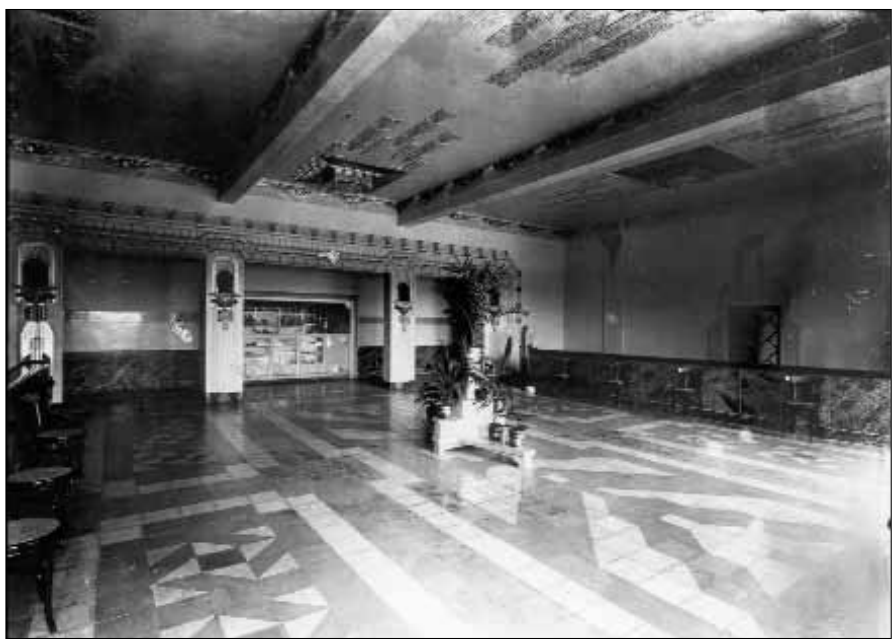




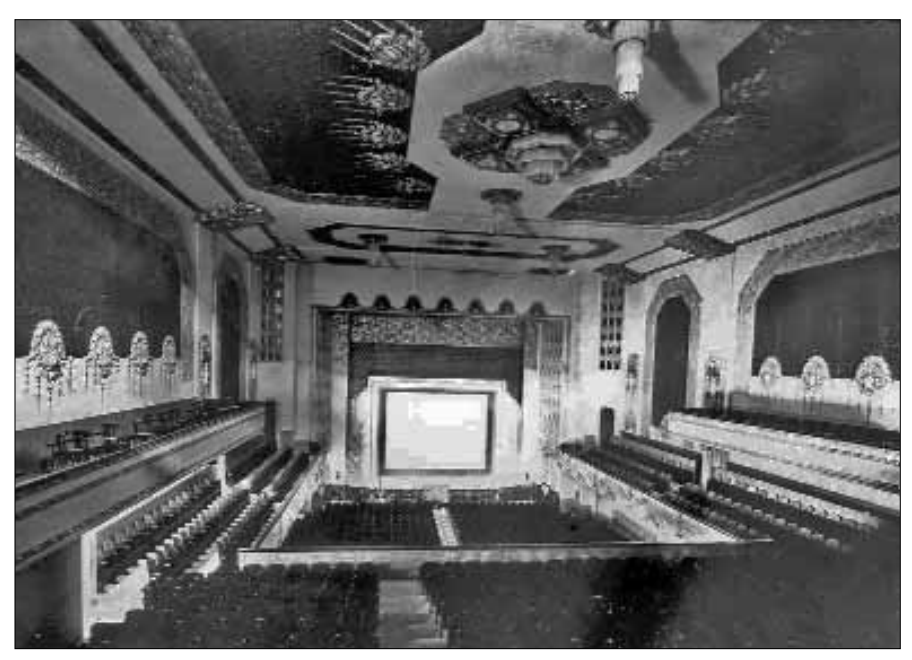

Fig. 11. Vista de la sala

interior y palcos, 1932

(ACM).

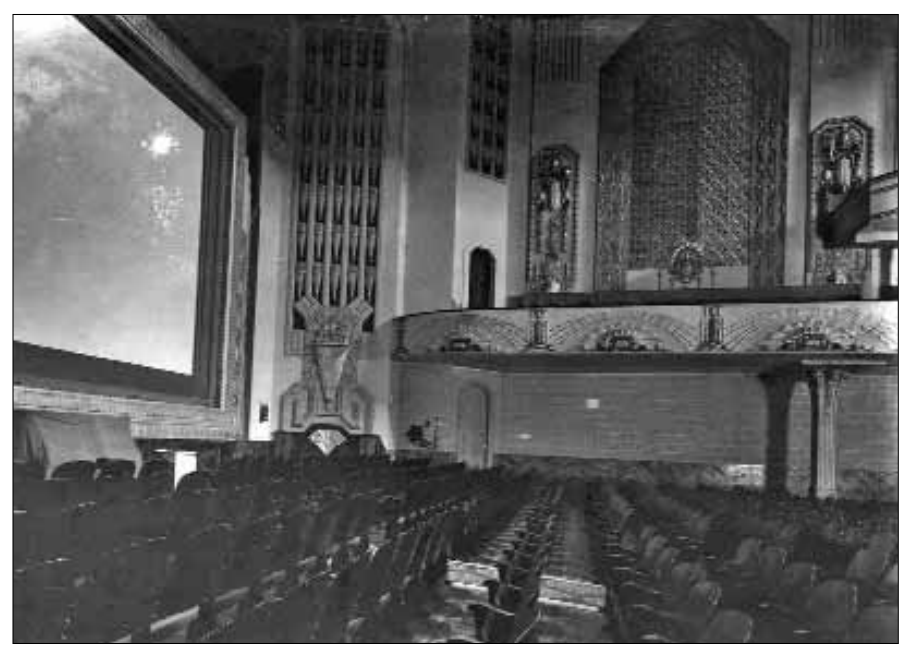

Fig. 12. Vista del interior, frontal de palco y pantalla, 1932 (ACM).

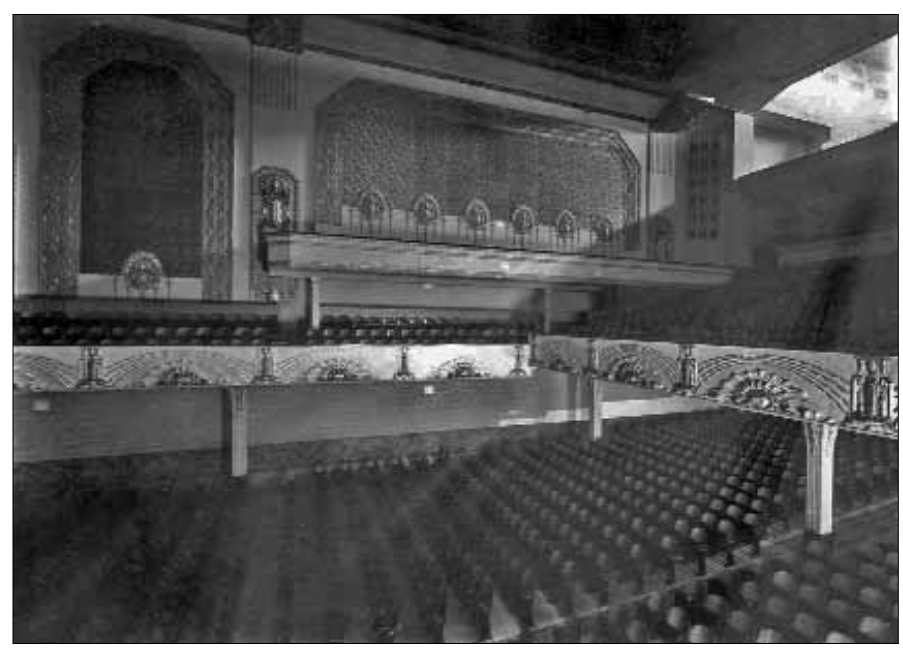

Fig. 13. Vista del interior, detalles decorativos y patio de butacas, 1932 (ACM). 
Fig. 14. Vista del cine desde la zona de pantalla, con patio de butacas y palcos, 1932

(ACM).

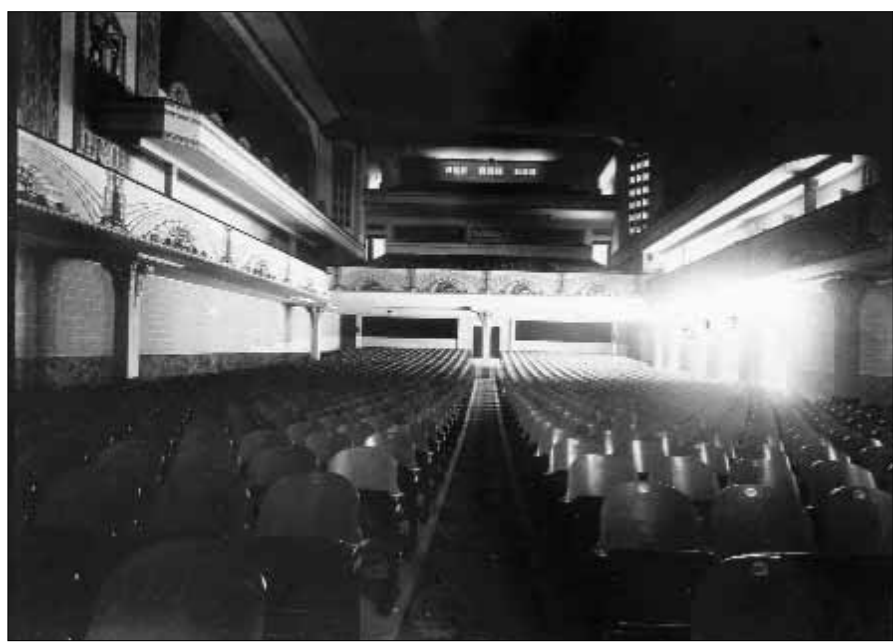

Fig. 15. D etalle de columna zona de butacas, 1932 (ACM).

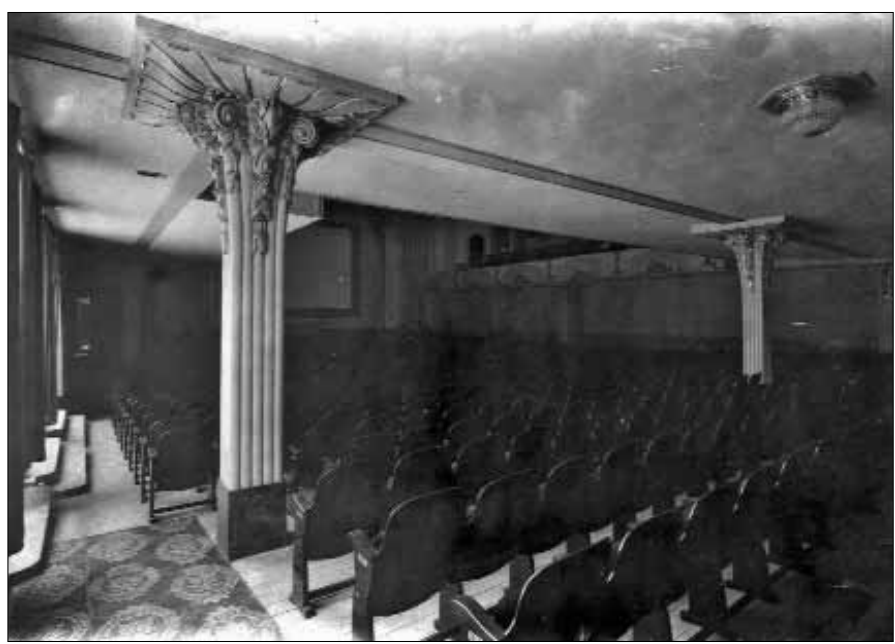




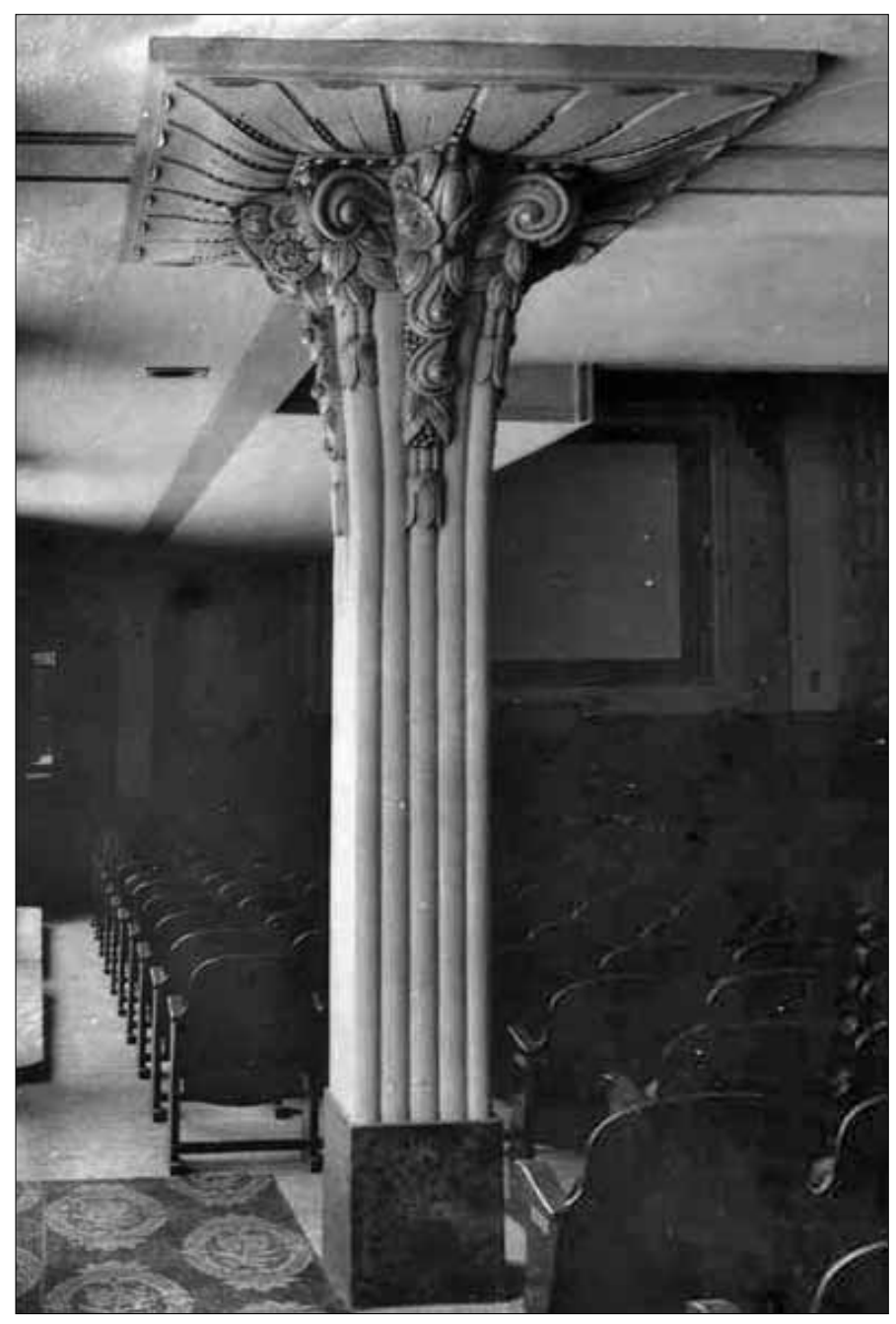

Fig. 16. Detalle de la

columna, 1932 (ACM).

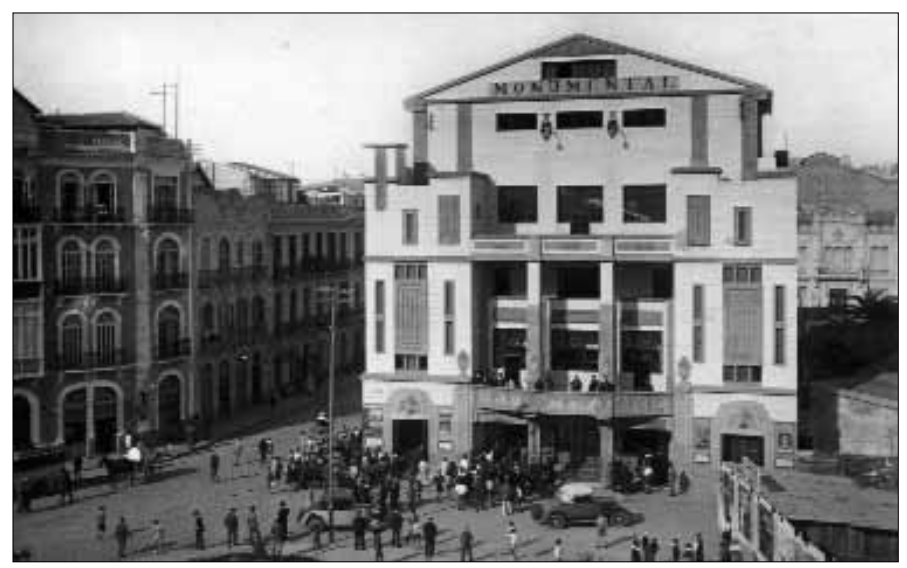

Fig. 17. Fachada principal del M onumental, años treinta 
Fig. 18. El M onumental desde la Plaza de España, primera mitad años treinta.

Fig. 19. Fachada principal y lateral del Monumental, segunda mitad años treinta.
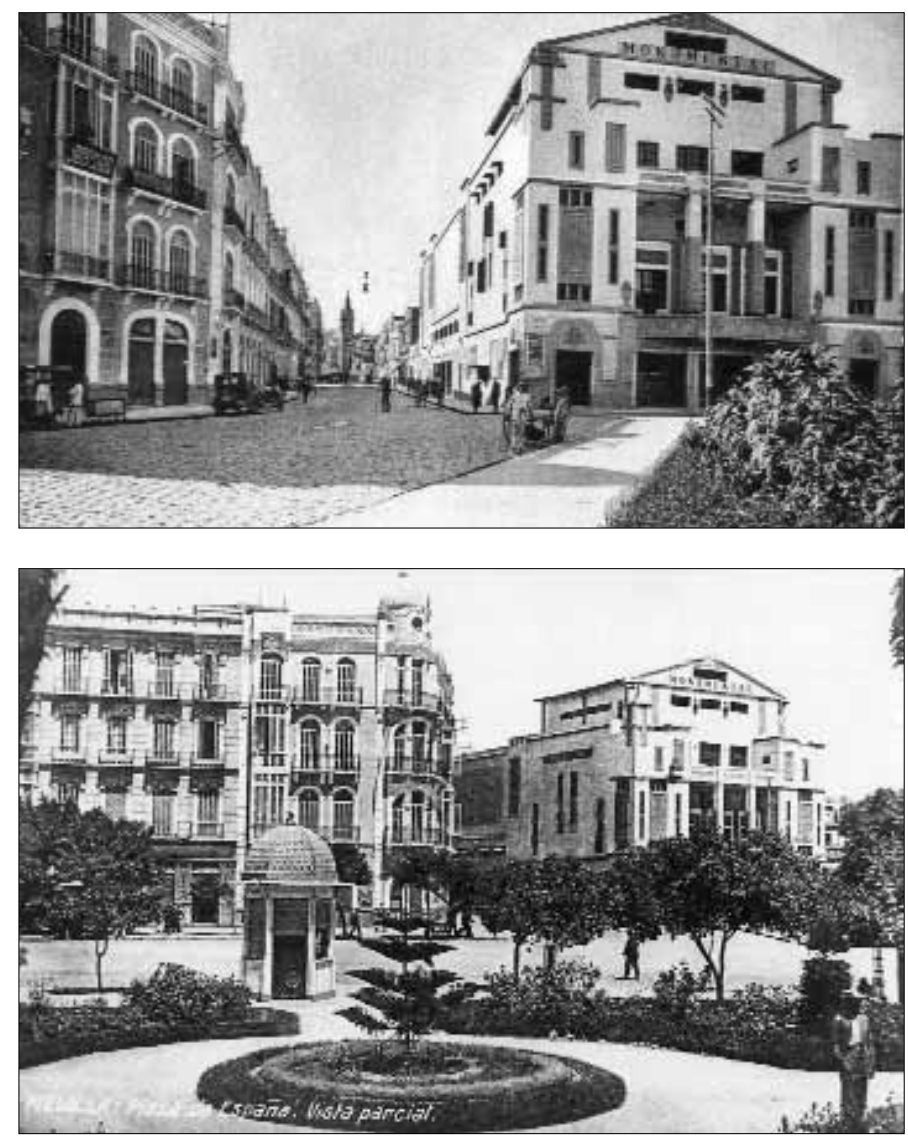

Fig. 20. Vista de las fachadas, años sesenta.

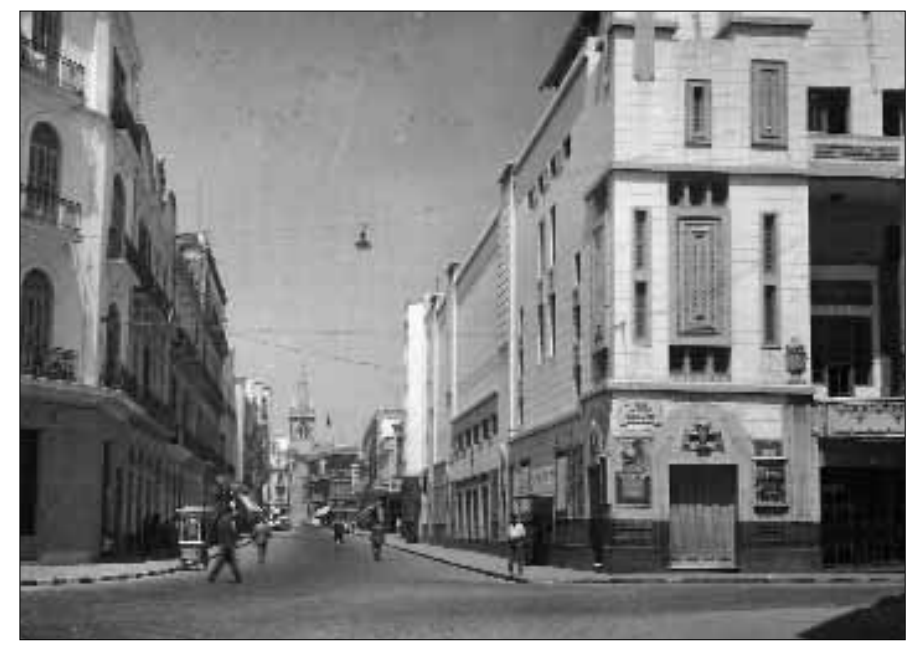




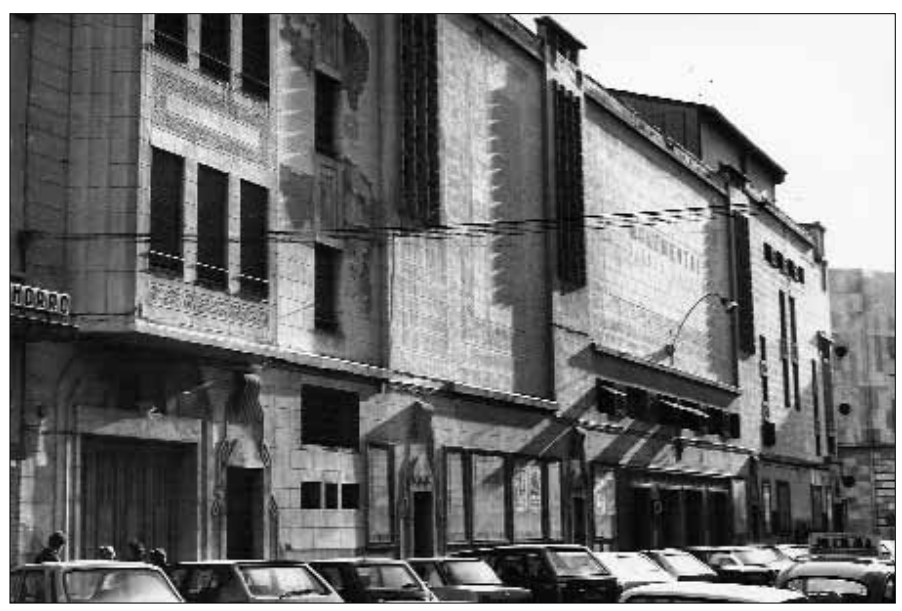

Fig. 21. Fachada lateral del cine en 1981.

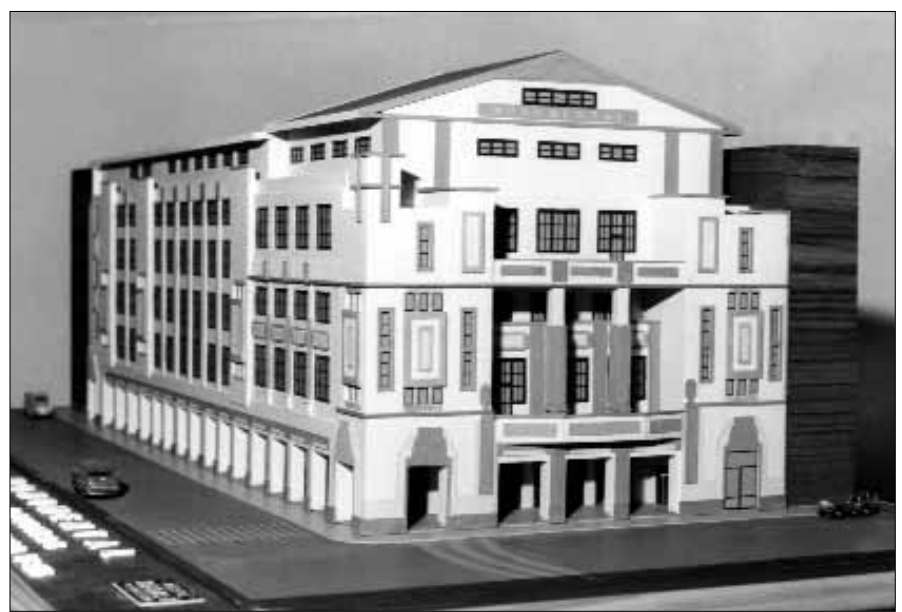

Fig. 22. Fotografía de la

maqueta de la transformación del cine, 1981.

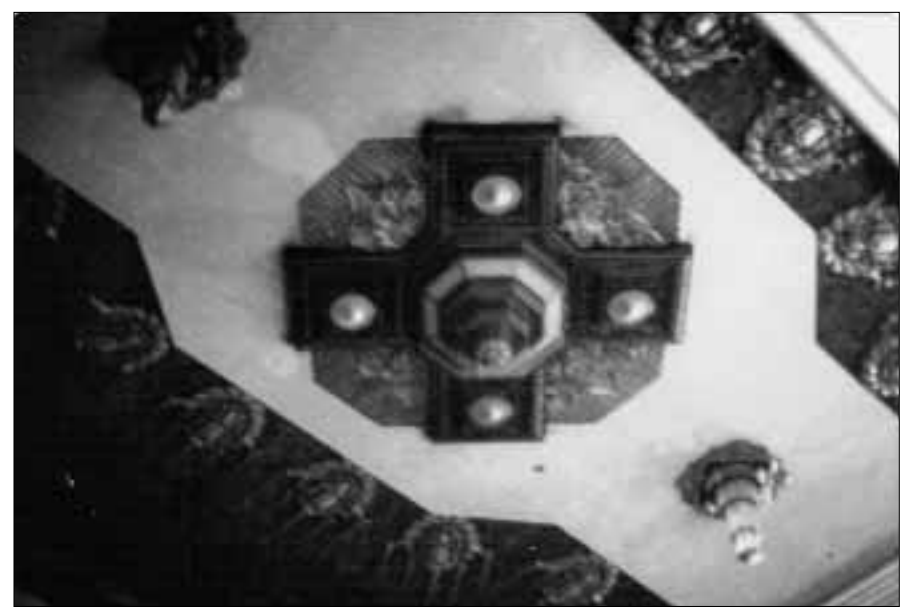

Fig. 23. Detalle de lámparas en el interior del cine, durante su destrucción, 1981 (fotografía de Jesús Miguel Sáez Cazorla, desde ahora JMSC). 
Fig. 24. Vista de la pared con restos del palco, durante su destrucción, 1981 (JMSC).

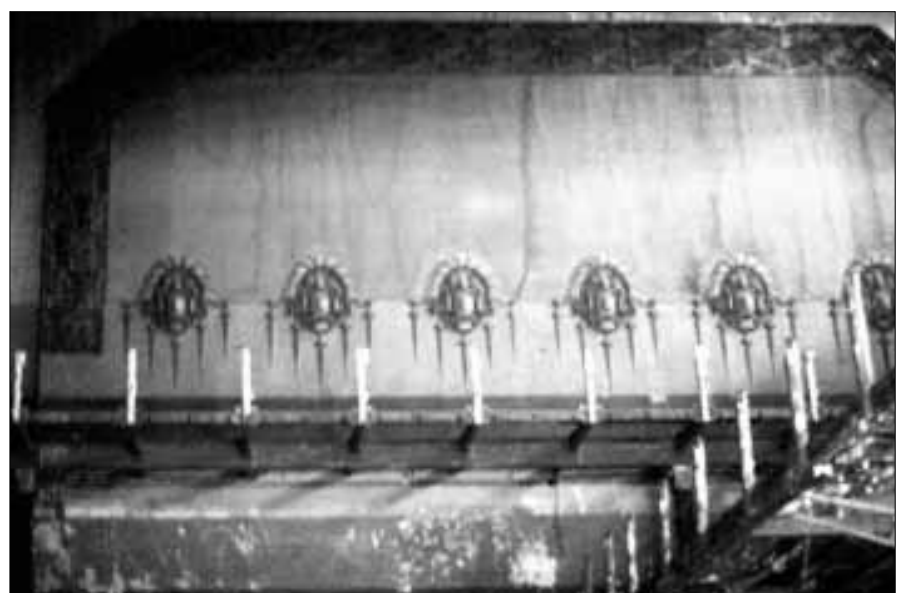

Fig. 25. Vista de elementos decorativos, durante su destrucción, 1981 (JMSC).

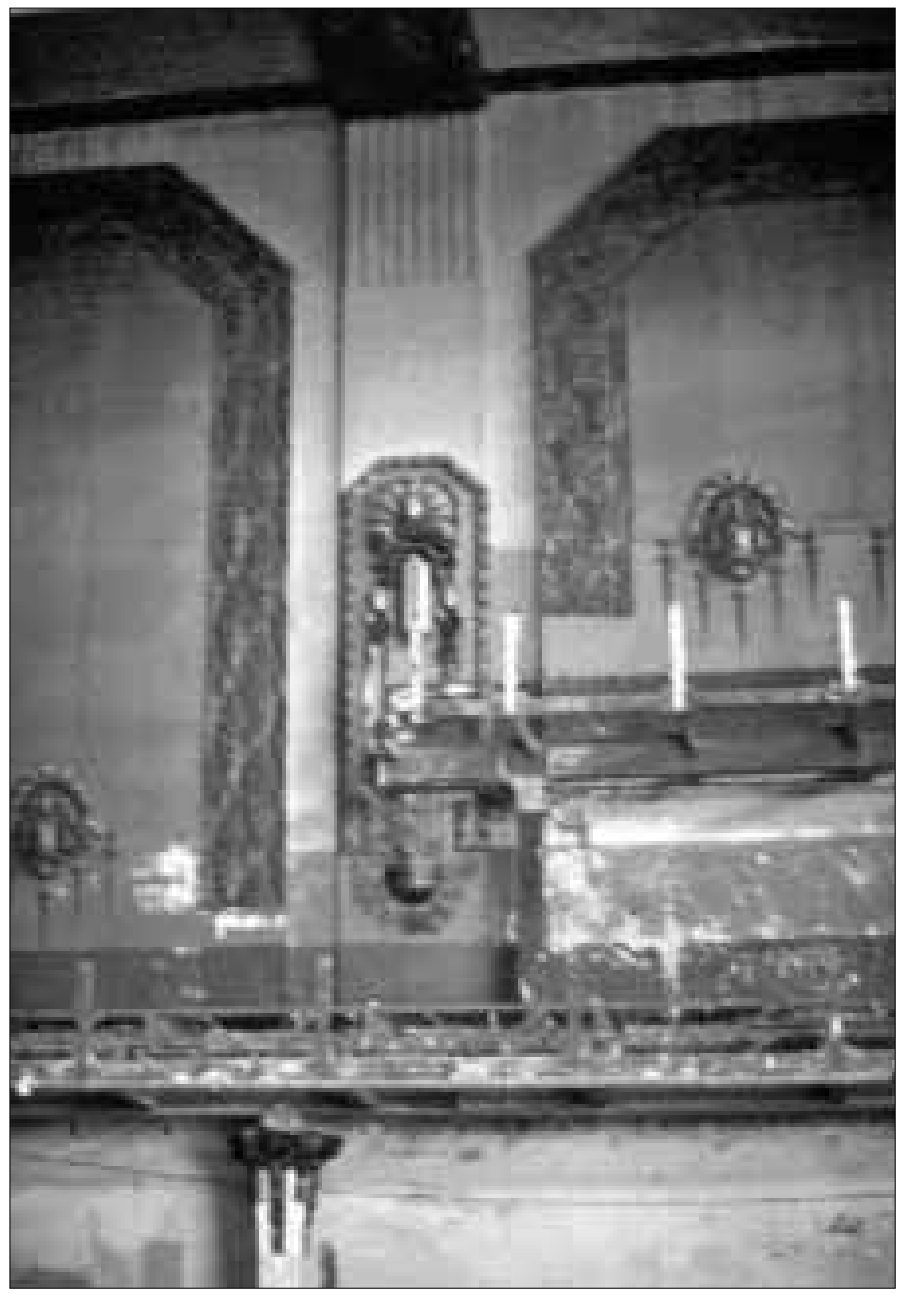




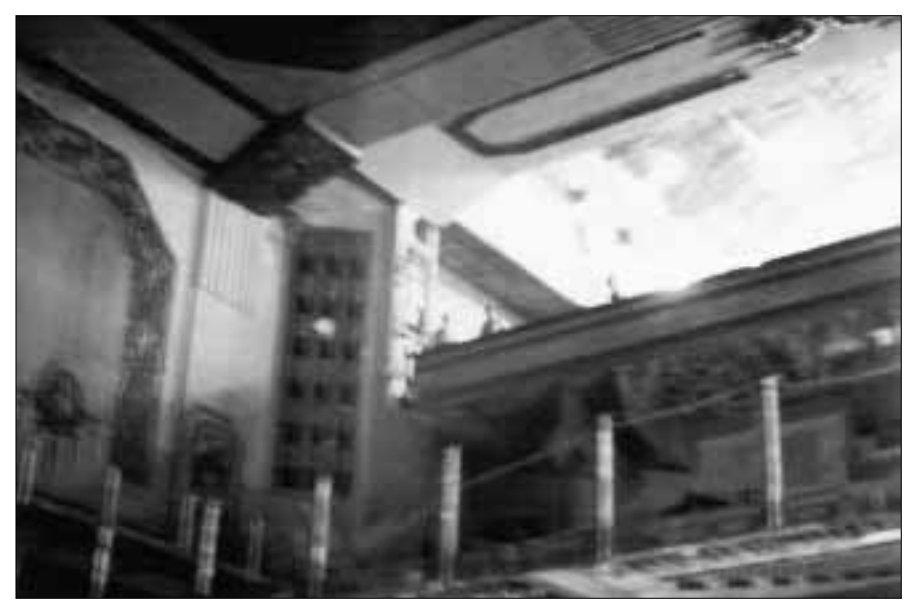

Fig. 26. Vista de la zona de palcos, durante su

destrucción, 1981 (JM SC).

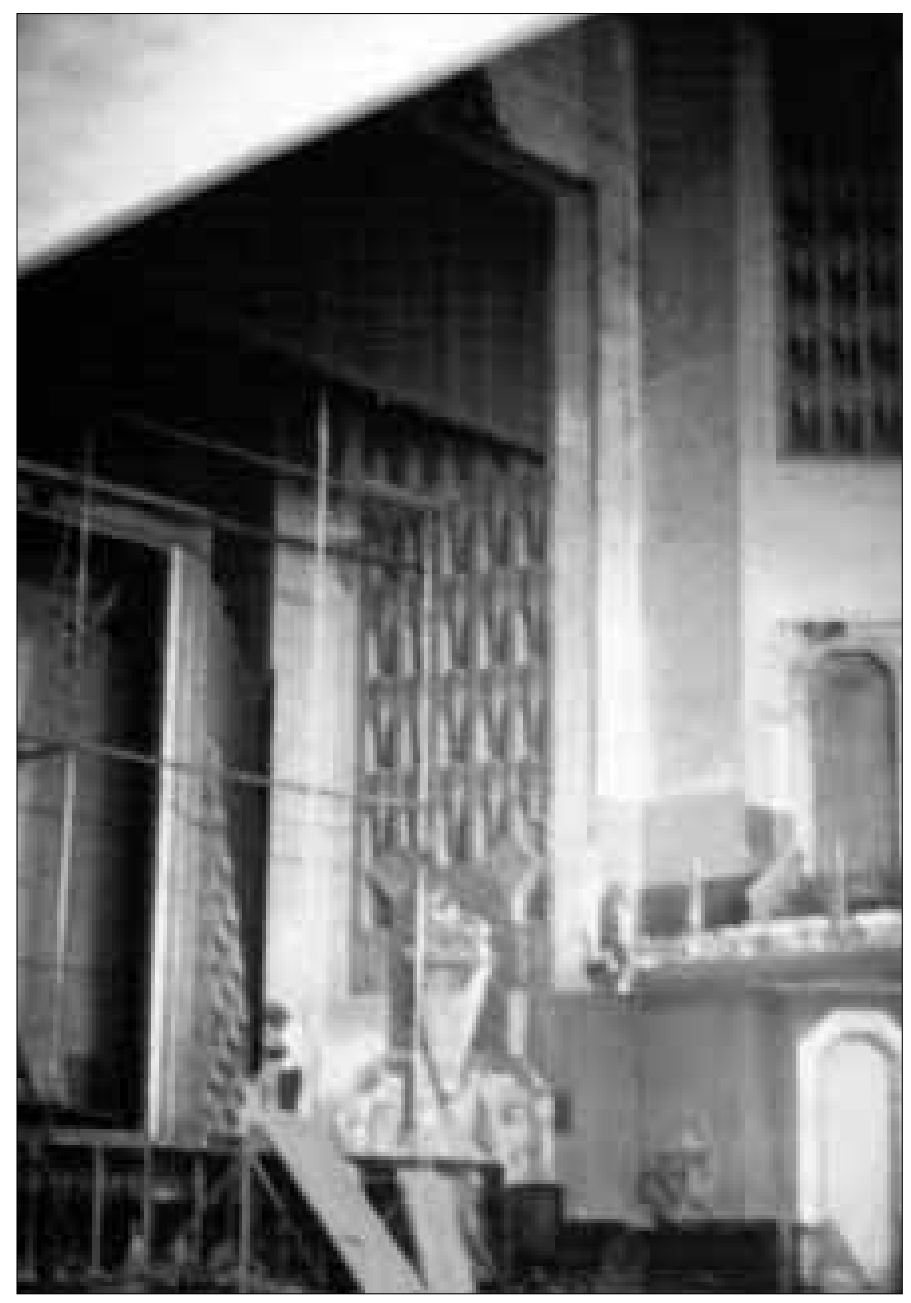

Fig. 27. Embocadura de la pantalla, durante su

destrucción, 1981 (JM SC). 
Fig. 28. Estado actual del edificio.

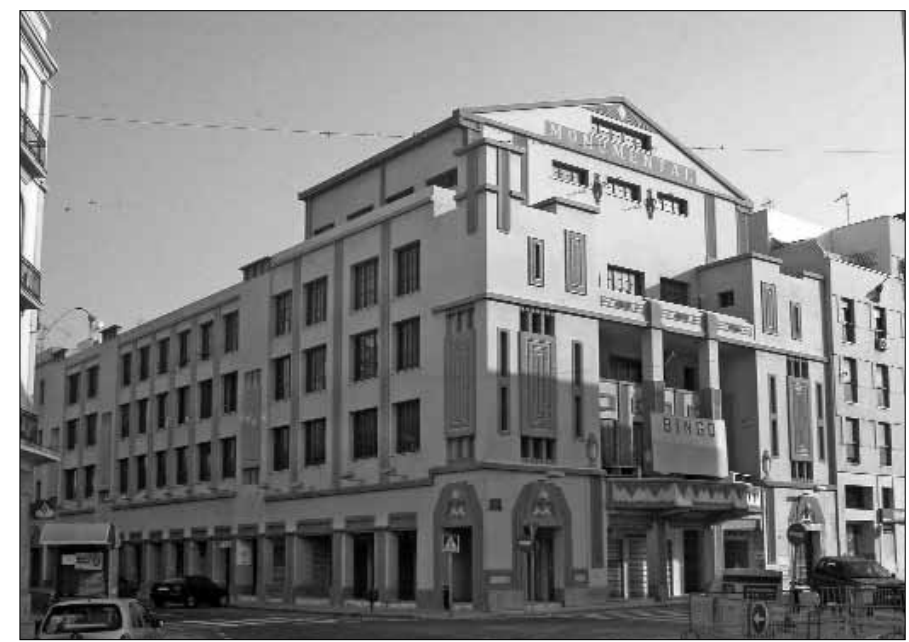

Fig. 29. Fachada principal en su estado actual.

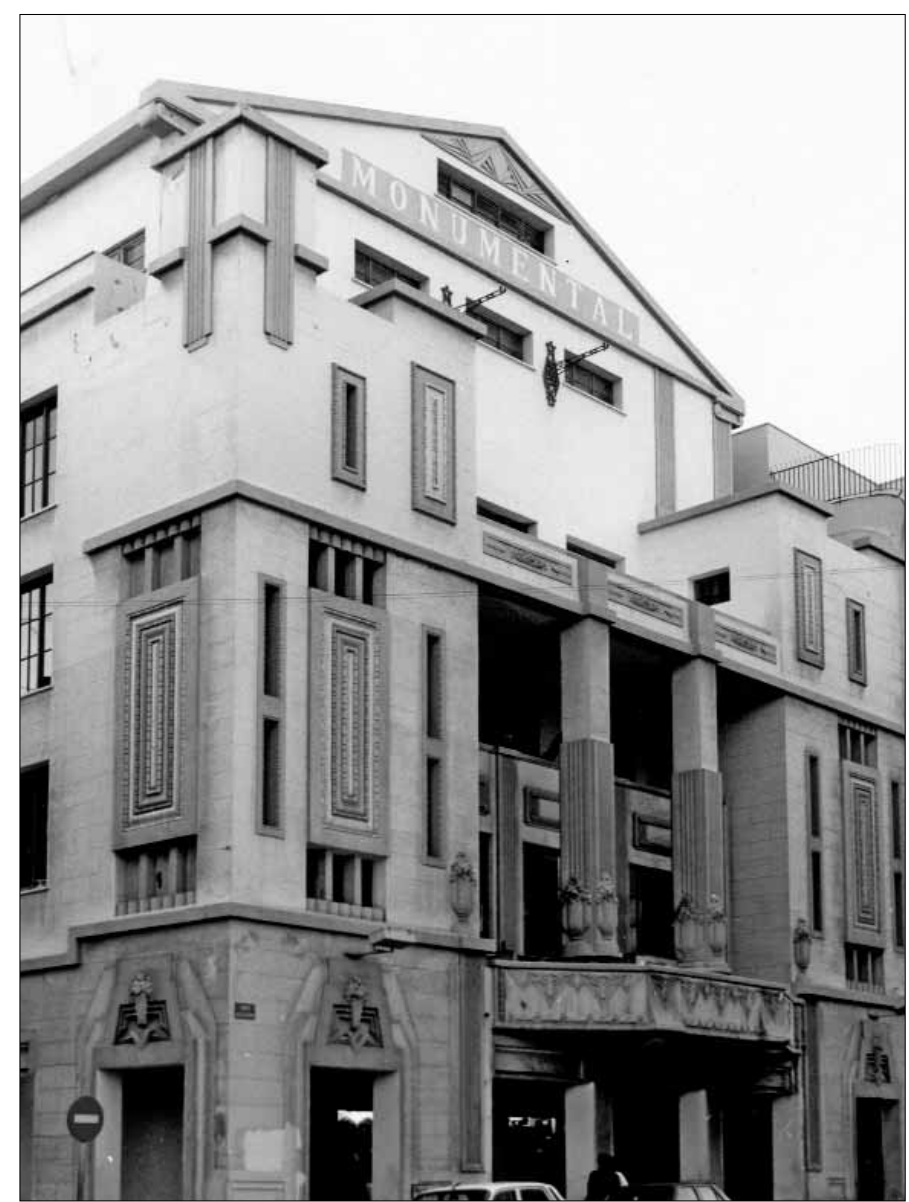




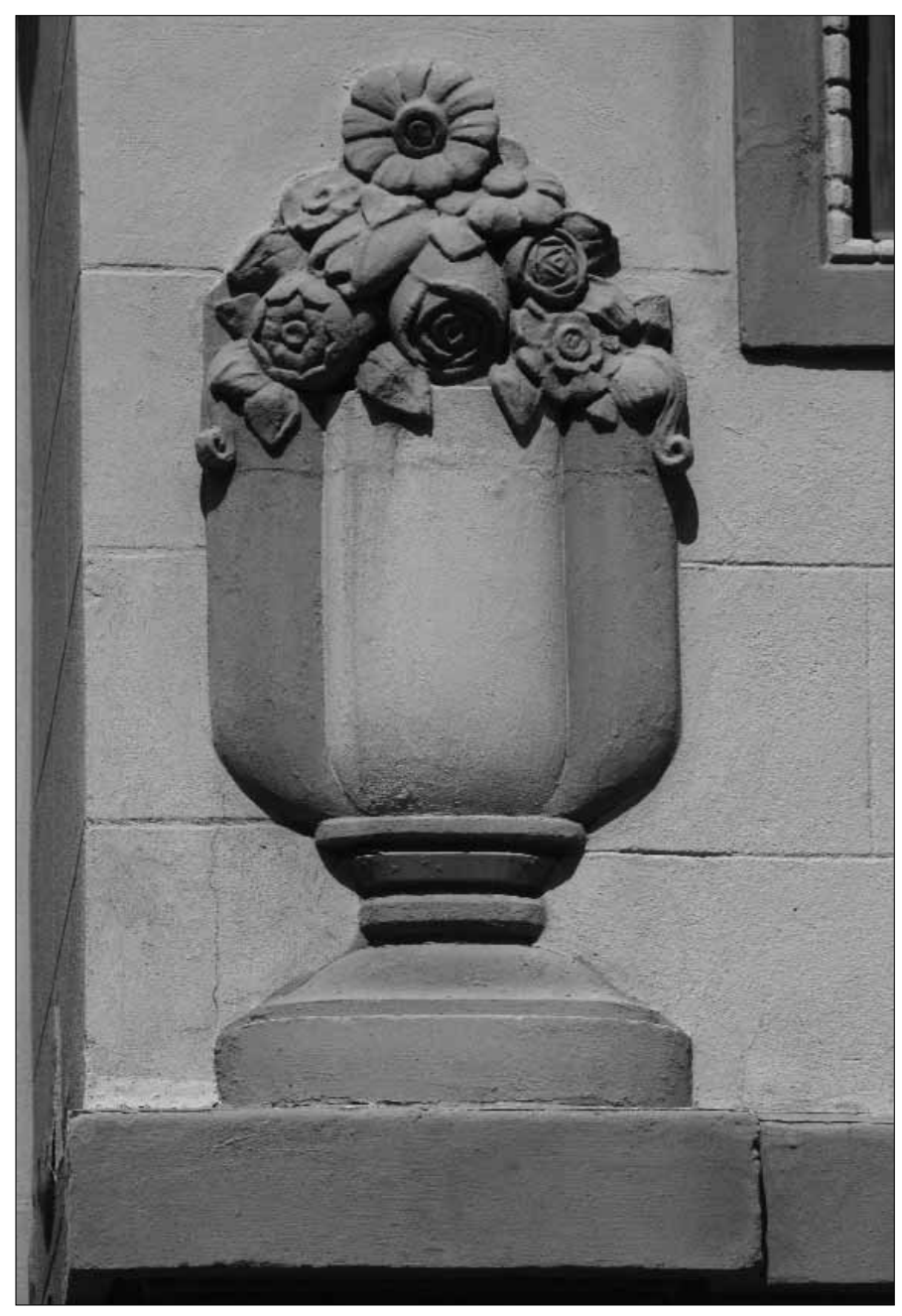

Fig. 30. Detalle decorativo de copa con flores, estado actual.

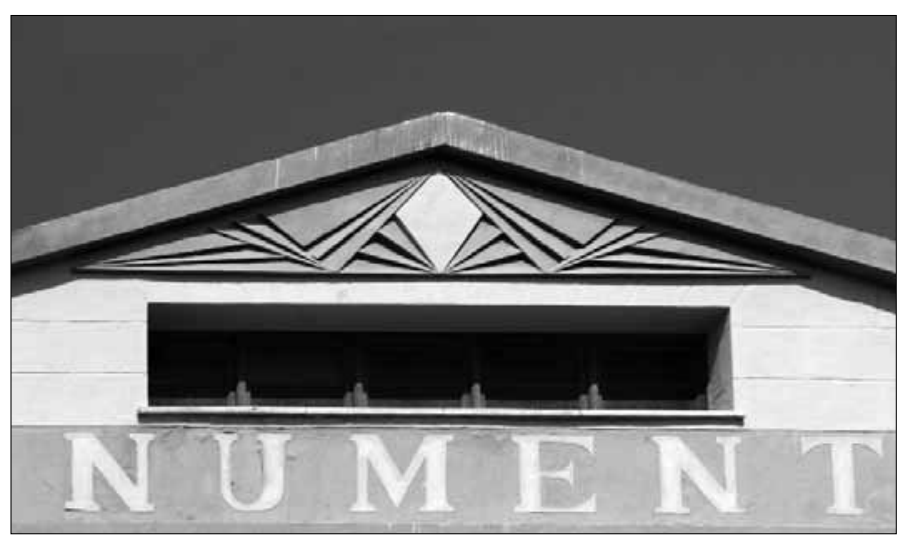

Fig. 31. Remate del frontón de fachada, estado actual. 
Fig. 32. Puerta con detalle ornamental, estado actual.

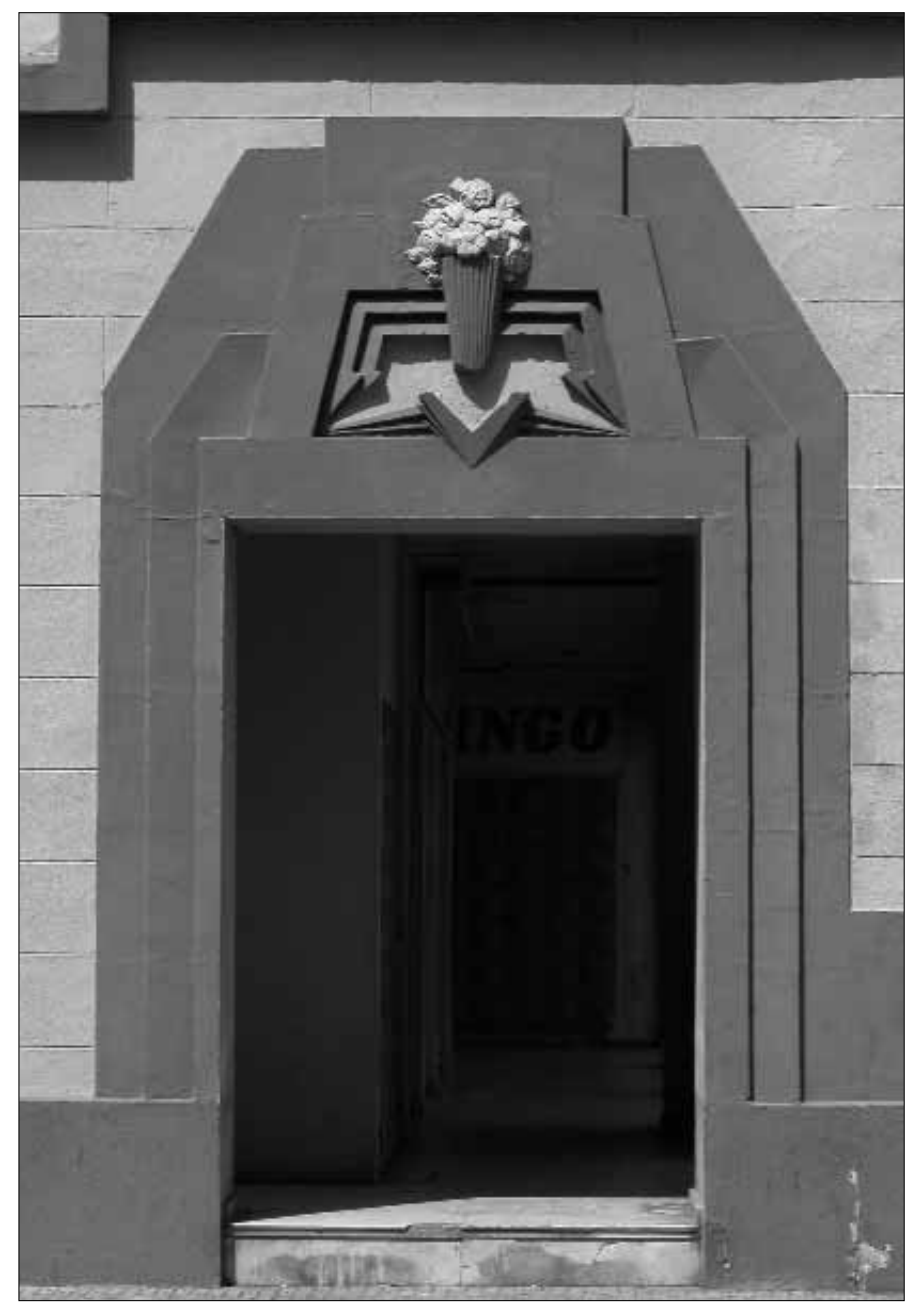

Fig. 33. Detalles ornamentales de fachada, estado actual.

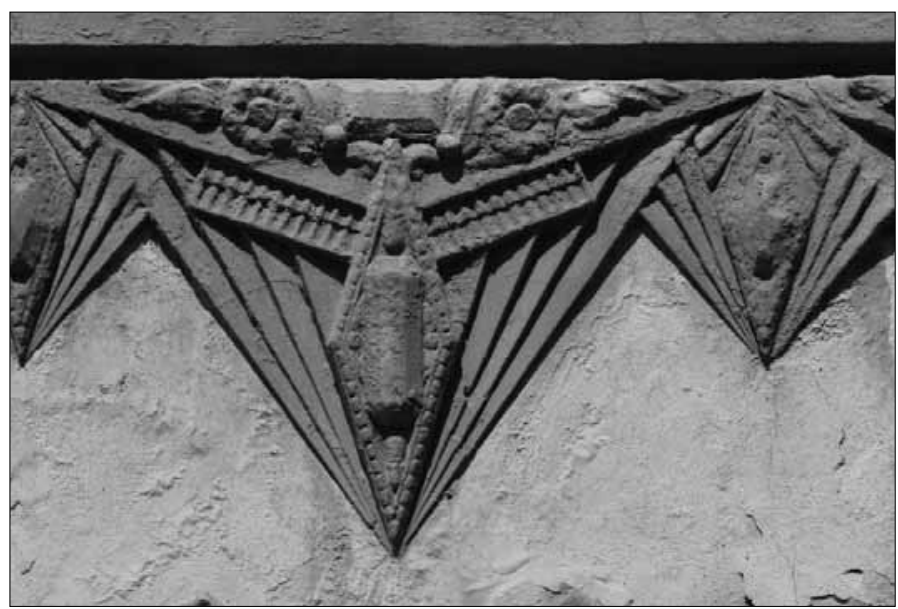




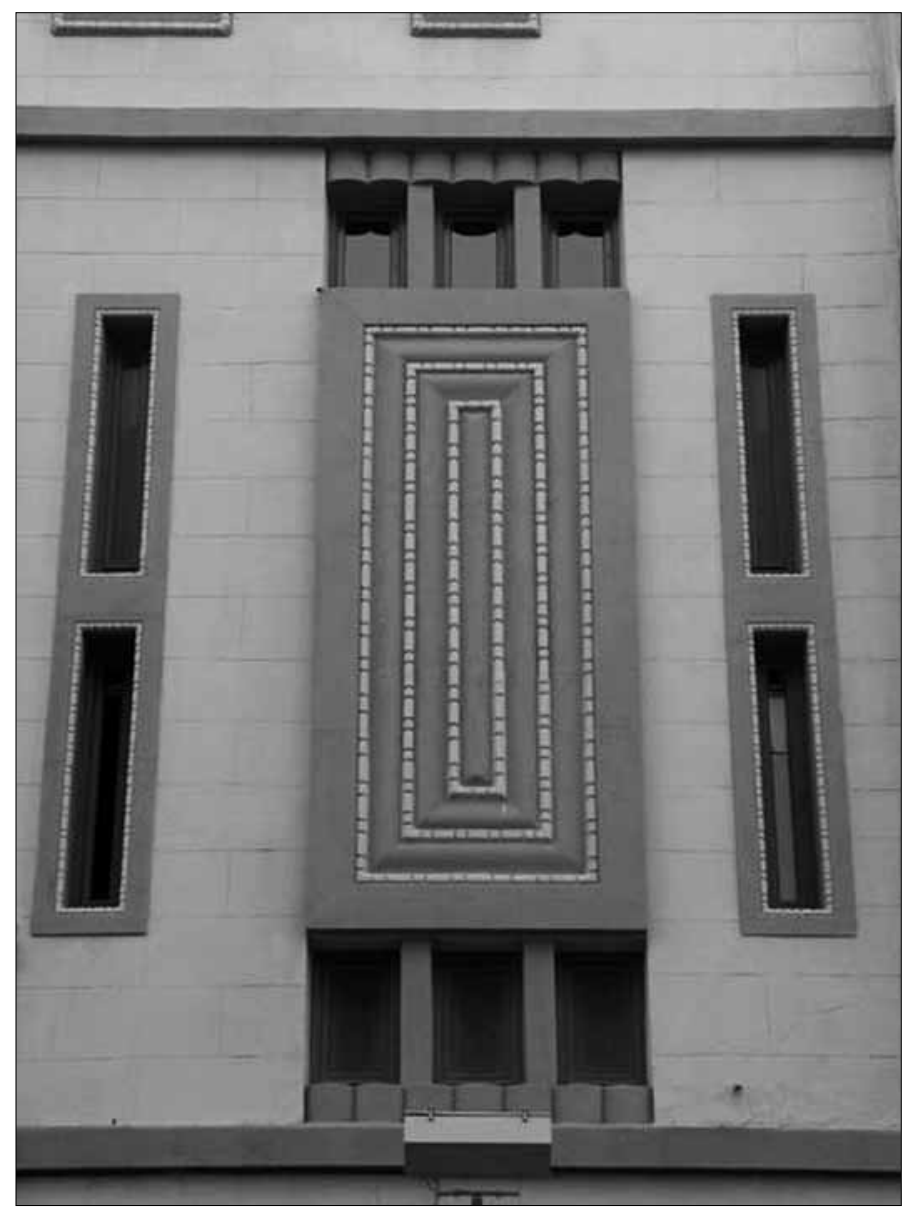

Fig. 34. Placas ornamentales, estado actual. 